CRYSTALLOGRAPHIC COMMUNICATIONS

ISSN 2056-9890

Received 22 November 2021

Accepted 25 November 2021

Edited by L. Van Meervelt, Katholieke Universiteit Leuven, Belgium

Keywords: crystal structure; dihydrofuranyl group (DHF); Hirshfeld surface analysis; dihydrofurylsilanes.

CCDC references: 2124286; 2124285

Supporting information: this article has supporting information at journals.iucr.org/e

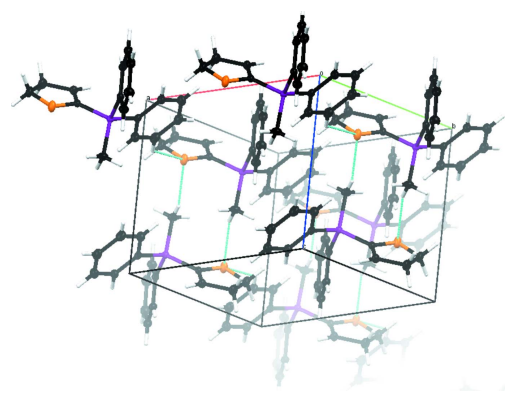

OPEN $\partial$ ACCESS

\section{Crystal structures and Hirshfeld surface analyses of bis(4,5-dihydrofuran-2-yl)dimethylsilane and (4,5-dihydrofuran-2-yl)(methyl)diphenylsilane}

\author{
Annika Schmidt, Anna Krupp, Eva Rebecca Barth and Carsten Strohmann*
}

Technische Universität Dortmund, Fakultät für Chemie und chemische Biologie, Anorganische Chemie, Otto-HahnStrasse 6, 44227 Dortmund, Germany. *Correspondence e-mail: carsten.strohmann@tu-dortmund.de

The title compounds, $\mathrm{C}_{10} \mathrm{H}_{16} \mathrm{O}_{2} \mathrm{Si}$ (1) and $\mathrm{C}_{17} \mathrm{H}_{18} \mathrm{OSi}$ (2), are classified as dihydrofurylsilanes, which show great potential as building blocks for various functionalized silanes. They both crystallize in the space group $P \overline{1}$ in the triclinic crystal system. Analyses of the Hirshfeld surfaces show packing-determining interactions for both compounds, resulting in a polymeric chain along the [011] for silane $\mathbf{1}$ and a layered-interconnected structure along the $b$-axis direction for silane 2.

\section{Chemical context}

Dihydrofurylsilanes are interesting starting materials for tailor-made silicon compounds. First presented in the 1980 s by Lukevics (Lukevics et al., 1985), they turned out to be versatile building blocks for multiple silicon compound classes. Tetrasubstituted silicon compounds are obtainable as a result of the excellent nature of the dihydrofuryl (DHF) substituent as a leaving group in various nucleophilic substitutions at the silicon atom. $\mathrm{Si}-\mathrm{C}(\mathrm{DHF})$ bond cleavages under substitution of the dihydrofuryl group was observed for the reactions with C-nucleophiles (e.g. organolithium compounds) (Gevorgyan et al., 1992), H-nucleophiles (e.g. $\mathrm{LiAlH}_{4}, \mathrm{NaH}, \mathrm{NaBH}_{4}$ ) (Gevorgyan et al., 1989, 1990), O-nucleophiles (e.g. $t$-butanol) and N-nucleophiles [e.g. $\mathrm{LiN}(\mathrm{Et})_{2}$ ] (Lukevics et al., 1997). By means of this efficient pathway, a noteworthy approach to pentacoordinated organyl silatranes has been made (Gevorgyan et al., 1997), as well as for ( $\alpha$-aminomethyl)silanes (Labrecque et al., 1994). Along with their easy preparation and hydrolytical and chromatographical stability (Gevorgyan et $a l ., 1997)$, dihydrofurylsilanes offer the potential to be useful reagents as protecting groups for the synthesis of aminomethylsilazanes (Colquhoun et al., 2011; Colquhoun \& Strohmann, 2012).<smiles>C[Si](C)(C1=CCCO1)C1=CCCO1</smiles>

1<smiles>C[Si](C1=CCCO1)(c1ccccc1)c1ccccc1</smiles>

2
Herein, we report the structures of two further dihydrofurylsilanes, bis(4,5-dihydrofuran-2-yl)dimethylsilane (1) and 
Table 1

Selected geometric parameters for compound $\mathbf{1}\left(\AA{ }^{\circ}\right)$.

\begin{tabular}{llll}
\hline Si1-C1 & $1.8742(3)$ & C1-Si1-C5 & $108.189(12)$ \\
Si1-C5 & $1.8693(3)$ & C1-Si1-C9 & $106.801(14)$ \\
Si1-C9 & $1.8631(3)$ & C1-Si1-C10 & $109.191(13)$ \\
Si1-C10 & $1.8579(3)$ & C5-Si1-C9 & $110.770(15)$ \\
& & C5-Si1-C10 & $108.128(13)$ \\
C1-C2 & $1.3370(4)$ & C9-Si1-C10 & $113.628(16)$ \\
C3-C4 & $1.5331(5)$ & & \\
C5-C6 & $1.3409(4)$ & & \\
C7-C8 & $1.5298(5)$ & & \\
\hline
\end{tabular}

(4,5-dihydrofuran-2-yl)(methyl)diphenylsilane (2) and their structural analysis, supplemented by a Hirshfeld surface analysis.

\section{Structural commentary}

The molecular structure of $\mathbf{1}$ is given in Fig. 1 and selected bond lengths and angles are given in Table 1. Compound $\mathbf{1}$ shows $C_{2}$ molecular symmetry. The lengths of the $\mathrm{Si}-\mathrm{C}(\mathrm{DHF})$ bonds are similar but slightly longer than the lengths of the $\mathrm{Si}-\mathrm{C}(\mathrm{Me})$ bonds. However, all bonds have characteristic dimensions (Allen et al., 1987). Furthermore, the length of the $\mathrm{C}=\mathrm{C}$ double bond corresponds well with literature values (Allen et al., 1987) and is clearly shortened in comparison to the $\mathrm{C}-\mathrm{C}$ single bonds in the dihydrofuranyl substituent. The silicon atom is tetrahedrally surrounded by its substituents, however slightly distorted as evident from the slight deviations from the ideal angle of $109.47^{\circ}$. These deviations are congruent with a former publication on dihydrofurylsilanes (Krupp et al., 2020). Both DHF planes display planarity while the $\mathrm{C} 1-\mathrm{C} 4 / \mathrm{O} 1$ ring has an r.m.s. deviation of $0.0197 \AA$ from an ideal least-squares plane with atom $\mathrm{C} 4$ showing the largest deviation of -0.0269 (2) $\AA$. The $\mathrm{C} 5-\mathrm{C} 8 / \mathrm{O} 2$ ring deviates more strongly from an ideal least-square plane with an r.m.s.

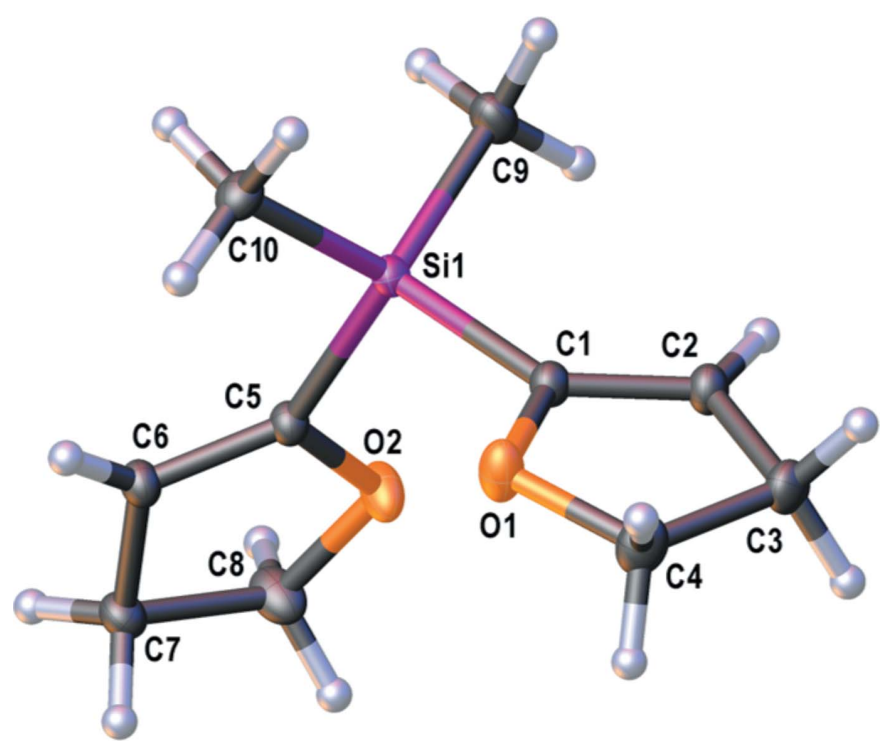

Figure 1

The molecular structure of compound $\mathbf{1}$ with displacement ellipsoids drawn at the $50 \%$ probability level.
Table 2

Selected geometric parameters for compound $\mathbf{2}\left(\AA{ }^{\circ}\right)$.

\begin{tabular}{llll}
\hline Si1-C1 & $1.8742(10)$ & C1-Si1-C5 & $108.44(4)$ \\
Si1-C5 & $1.8721(9)$ & C1-Si1-C11 & $105.51(4)$ \\
Si1-C11 & $1.8713(10)$ & C1-Si1-C17 & $109.26(5)$ \\
Si1-C17 & $1.8591(11)$ & C5-Si1-C11 & $113.08(4)$ \\
& & C5-Si1-C17 & $110.49(5)$ \\
C1-C2 & $1.3356(14)$ & C11-Si1-C17 & $109.88(59$ \\
C3-C4 & $1.5416(17)$ & & \\
\hline
\end{tabular}

deviation of $0.0608 \AA$, with the $\mathrm{C} 8$ atom deviating the most by 0.0838 (2) $\AA$. The angle between the normals of the leastsquares planes through the DHF rings is $78.943(15)^{\circ}$.

The molecular structure of $\mathbf{2}$ is given in Fig. 2 and selected bond lengths and angles are given in Table 2. The length of the $\mathrm{Si}-\mathrm{C}(\mathrm{DHF})$ bond is in the range of the lengths of the $\mathrm{Si}-$ $\mathrm{C}(\mathrm{Ph})$ bonds, which are again slightly longer than the $\mathrm{Si}-$ $\mathrm{C}(\mathrm{Me})$ bond. The bond lengths of the dihydrofuran ring are consistent with those of structure 1. Again, a slightly distorted tetrahedral environment at the silicon atom is observed. The DHF ring is less planar than the phenyl rings, with an r.m.s. deviation from the least-squares plane of $0.0426 \AA$ with the C4 atom having the largest deviation of -0.0582 (7) $\AA$. The phenyl rings show r.m.s. deviations of 0.0066 and $0.0047 \AA$. The angle between the normals of the least-squares planes of the DHF ring and the C5-C10 phenyl ring is $87.68(4)^{\circ}$ and the angle between the normals of the least-squares planes of the phenyl rings is $60.03(4)^{\circ}$.

\section{Supramolecular features}

The crystal packing of compound $\mathbf{1}$ is defined by $\mathrm{C} 10-$ $\mathrm{H} 10 A \cdots \mathrm{C} 2{ }^{\mathrm{i}}$ van der Waals interactions as can be seen in Fig. 3. The interactions show relatively large distances $\left[\mathrm{C} 10 \cdots \mathrm{C} 2^{\mathrm{i}}=\right.$ $3.6208(5) \AA, \quad \mathrm{H} 10 A \cdots \mathrm{C} 2^{\mathrm{i}}=2.752(10) \AA$ and $\mathrm{C} 10-$

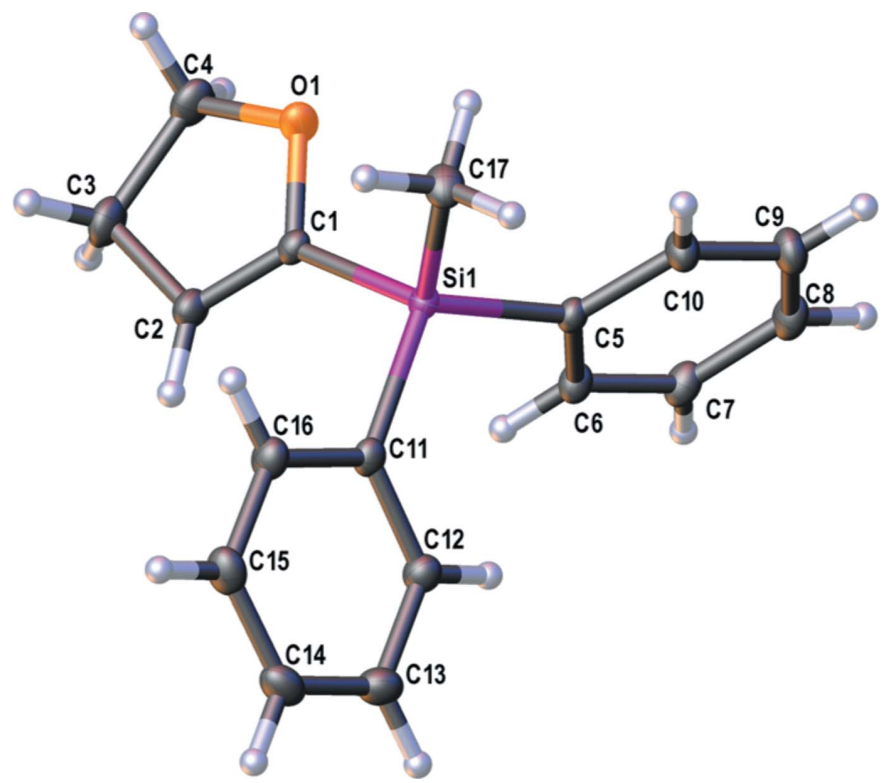

Figure 2

The molecular structure of compound 2 with displacement ellipsoids drawn at the $50 \%$ probability level. 


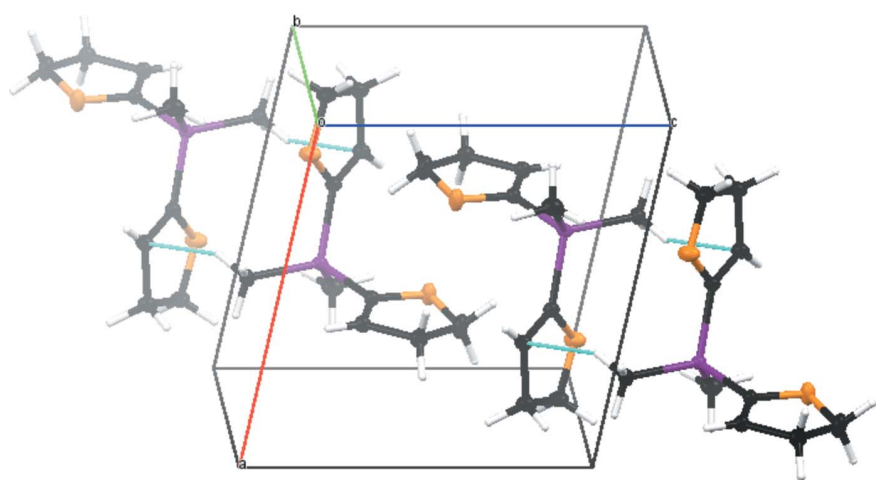

Figure 3

The crystal packing of compound 1 . C $-\mathrm{H} \cdots \mathrm{C}$ van der Waals interactions are shown as dashed lines.

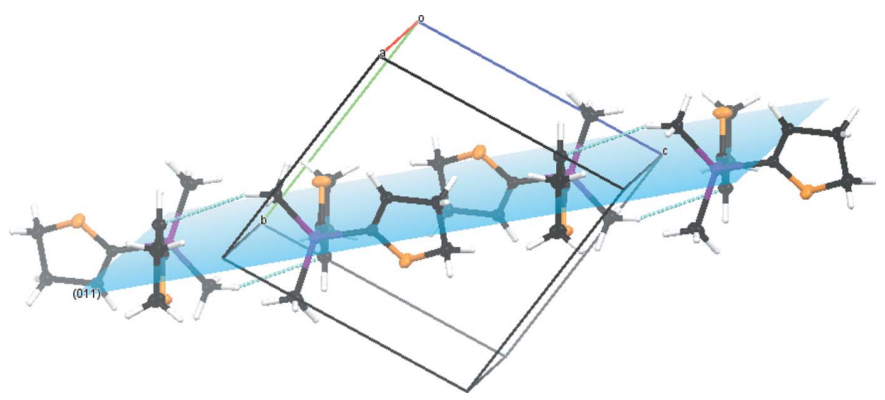

Figure 4

The crystal packing of compound $\mathbf{1}$ with the (011) plane shaded in blue. $\mathrm{C}-\mathrm{H} \cdots \mathrm{C}$ van der Waals interactions are shown as dashed lines.

$\mathrm{H} 10 A \cdots \mathrm{C} 2^{\mathrm{i}}=148.4(8)^{\circ}$; symmetry code: (i) $-x+1,-y+2$, $-z$ ]. As a result of the interactions between carbon atom $\mathrm{C} 2$ and hydrogen atom $\mathrm{H} 10 A$, a polymeric chain structure along the [011] direction is formed (Fig. 4). The interactions can be displayed by a Hirshfeld surface analysis (Spackman \& Jayatilaka, 2009) generated by CrystalExplorer21 (Spackman et al., 2021), here indicated by the red spots (Fig. 5). The Hirshfeld surface mapped over $d_{\text {norm }}$ is in the range from -0.0783 to 1.0981 a.u. The contributions of the different types of intermolecular interactions for $\mathbf{1}$ are shown in the two-dimensional

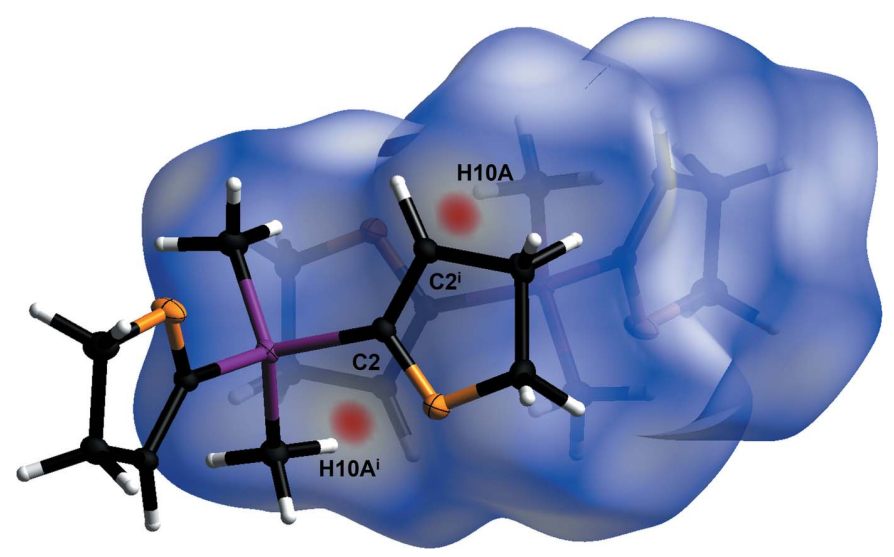

Figure 5

Hirshfeld surface analysis of $\mathbf{1}$ showing close contacts in the crystal. The van der Waals interaction between carbon atom $\mathrm{C} 2$ and the $\mathrm{H} 10 A$ hydrogen atom is labeled. [Symmetry code: (i) $-x+1,-y+2,-z$ ].
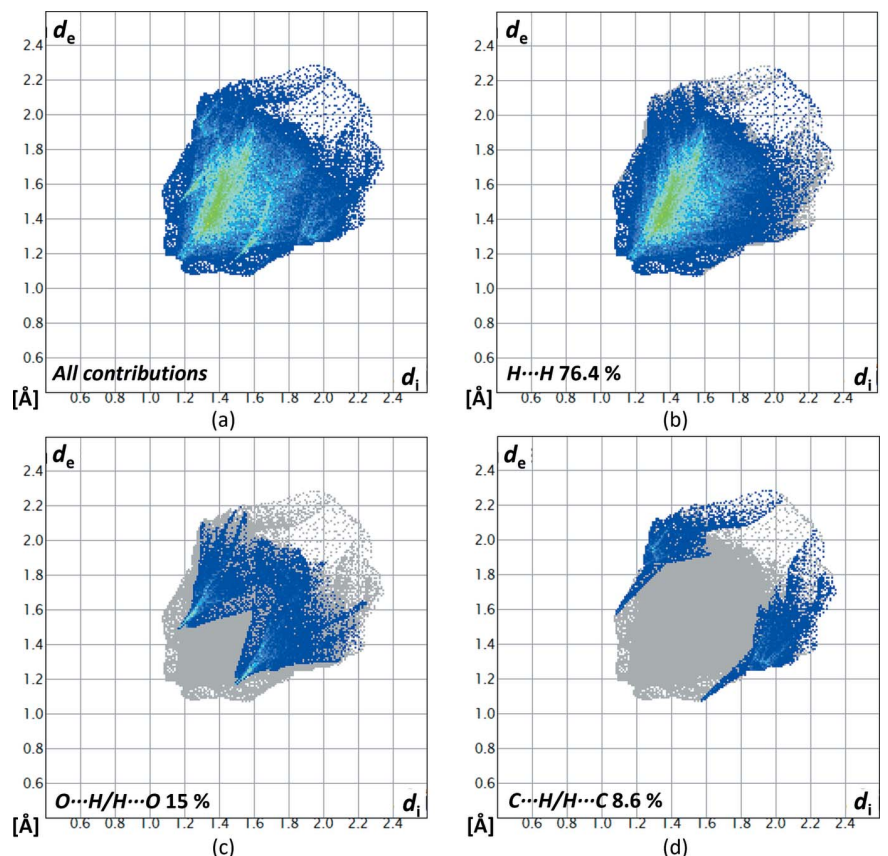

Figure 6

(d)

Two-dimensional fingerprint plots for compound 1, showing (a) all contributions, and $(b)-(d)$ delineated showing the contributions of atoms within specific interacting pairs (blue areas).

fingerprint plots (McKinnon et al., 2007) in Fig. 6. The contribution of the $\mathrm{H} \cdots \mathrm{H}$ interactions, with a value of $76.4 \%$, has the largest share of the crystal packing of $\mathbf{1}$. The $\mathrm{O} \cdots \mathrm{H} /$ $\mathrm{H} \cdots \mathrm{O}$ interactions have a smaller share with a $15 \%$ contribution and the $\mathrm{C} \cdots \mathrm{H} / \mathrm{H} \cdots \mathrm{C}$ interactions with a $8.6 \%$ contribution. Both heteronuclear interactions appear as spikes.

The structure of compound $\mathbf{2}$ is more strongly defined by $\mathrm{C}-\mathrm{H} \cdots \mathrm{O}$ hydrogen bonds (Fig. 7, Table 3). Two different layers are formed along the $b$-axis direction and interconnected by hydrogen bonds between the $\mathrm{O} 1$ atom and the $\mathrm{H} 17 \mathrm{C}$ atom. An additional interaction in each of the layers is observed by $\mathrm{C}-\mathrm{H}$. . O hydrogen bonds between the $\mathrm{O} 1$ atom

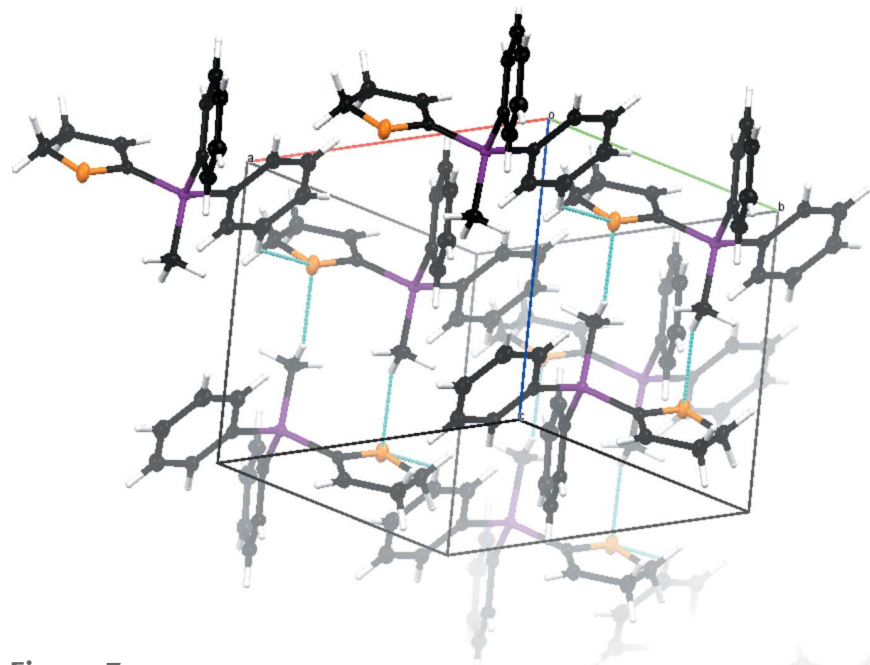

Figure 7

The crystal packing of compound 2. $\mathrm{C}-\mathrm{H} \cdots \mathrm{O}$ hydrogen bonds are shown as dashed lines. 
Table 3

Hydrogen-bond geometry $\left(\AA{ }^{\circ}\right)$ for $\mathbf{2}$.

\begin{tabular}{lllll}
\hline$D-\mathrm{H} \cdots A$ & $D-\mathrm{H}$ & $\mathrm{H} \cdots A$ & $D \cdots A$ & $D-\mathrm{H} \cdots A$ \\
\hline $\mathrm{C} 15-\mathrm{H} 15 \cdots \mathrm{O} 1^{\mathrm{i}}$ & $0.969(16)$ & $2.640(16)$ & $3.3422(13)$ & $129.6(12)$ \\
$\mathrm{C}^{\mathrm{ii}} 17 \mathrm{H} 17 C \cdots \mathrm{O} 1^{\mathrm{ii}}$ & $0.992(19)$ & $2.584(19)$ & $3.5168(14)$ & $156.5(15)$ \\
\hline
\end{tabular}

Symmetry codes: (i) $x, y+1, z$; (ii) $-x+2,-y+1,-z+1$.

and the $\mathrm{H} 15$ atom. The $\mathrm{C} 17-\mathrm{H} 17 \mathrm{C} \cdots \mathrm{O} 1^{\mathrm{ii}}$ hydrogen bond can be described by the $R_{2}^{2}(10)$ graph-set motif and the $\mathrm{C} 15-$ H15 . . O $1^{\mathrm{i}}$ hydrogen bond by the $C_{1}^{1}(7)$ graph-set motif (Etter et al., 1990). Both $\mathrm{C}-\mathrm{H}$. . O hydrogen bonds can be identified as weak interactions according to Desiraju \& Steiner (2001). A Hirshfeld analysis, carried out analogously as for structure 1, was used to further study the crystal packing. In Fig. 8, the nearest contacts are shown in red. The Hirshfeld surface mapped over $d_{\text {norm }}$ is in the range from -0.0717 to 1.0768 a.u. By analysis of the two-dimensional fingerprint plots (Fig. 9), again, the biggest contribution to the crystal packing can be assigned to $\mathrm{H} \cdot \mathrm{H}$ interactions $(66 \%)$. Although the closest contacts were identified as $\mathrm{C}-\mathrm{H} \cdots \mathrm{O}$ hydrogen bonds, $\mathrm{O} \cdots \mathrm{H} /$ $\mathrm{H} \cdots \mathrm{O}$ interactions contribute only $6.4 \%$ to the crystal

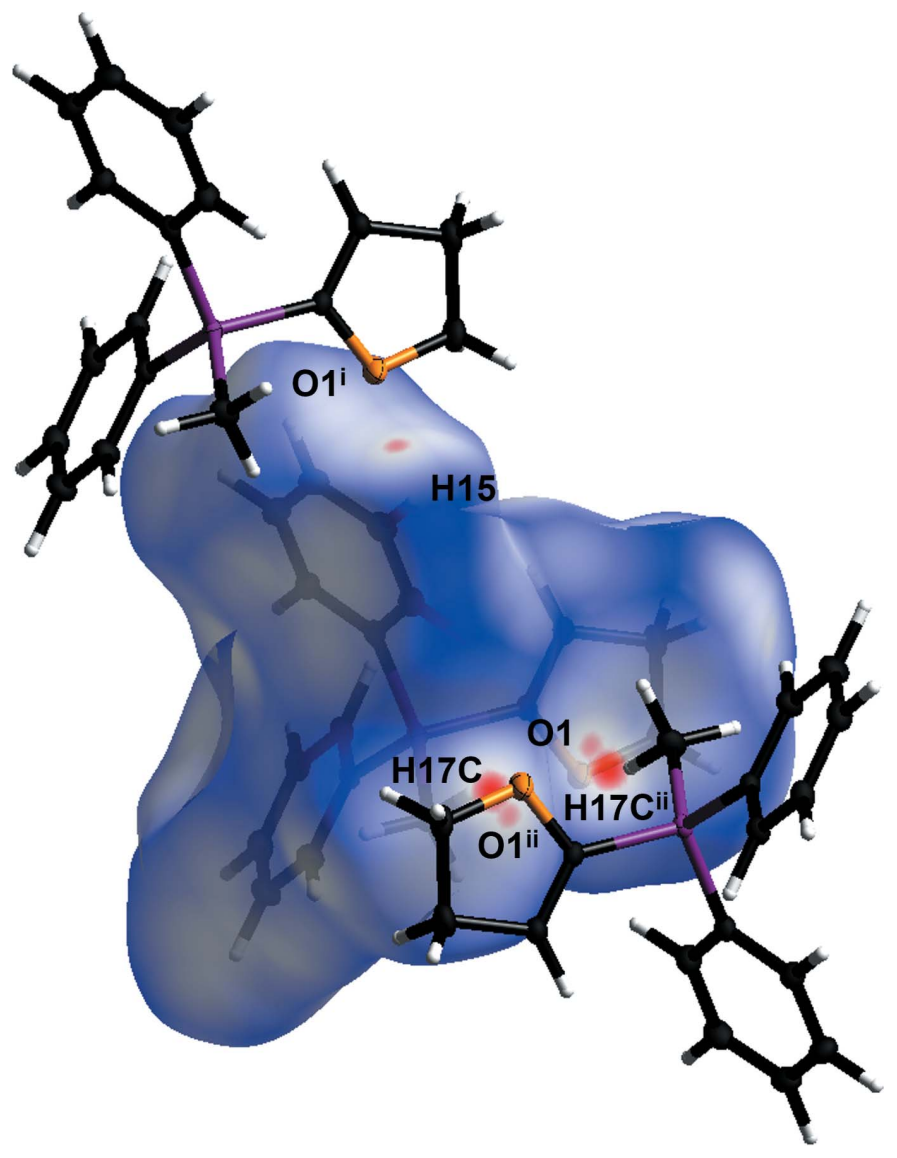

Figure 8

Hirshfeld surface analysis of $\mathbf{2}$ showing close contacts in the crystal. The weak hydrogen bond between oxygen atom $\mathrm{O} 1$ and the $\mathrm{H} 15$ hydrogen atom, as well as the weak hydrogen bonds between the oxygen atom O1 and the $\mathrm{H} 17 \mathrm{C}$ hydrogen atom are labeled. [Symmetry codes: (i) $x, y+1, z$; (ii) $-x+2,-y+1,-z+1]$.

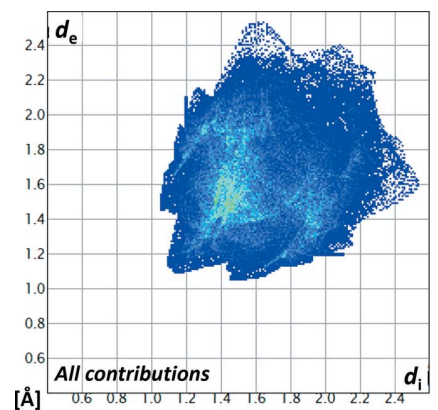

(a)

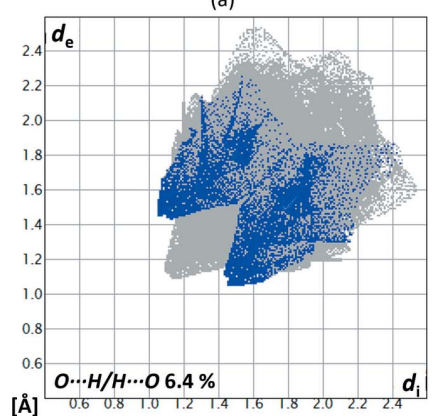

(c)

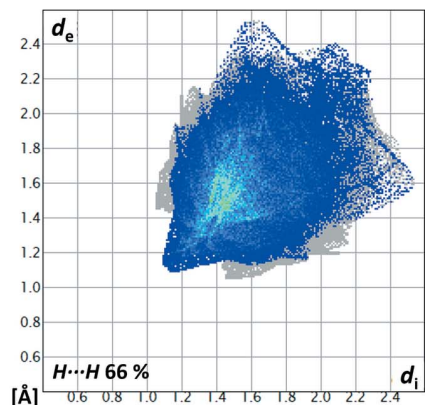

(b)

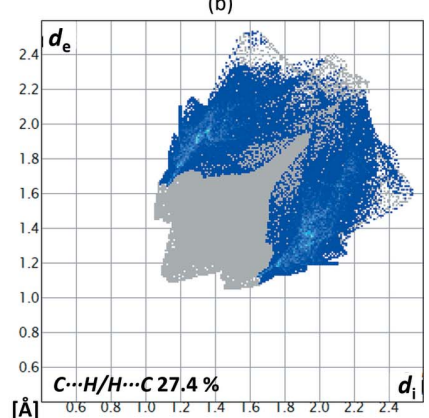

(d)

Figure 9

Two-dimensional fingerprint plots for compound 2, showing (a) all contributions, and $(b)-(d)$ delineated showing the contributions of atoms within specific interacting pairs (blue areas).

packing, while $\mathrm{C} \cdots \mathrm{H} / \mathrm{H} \cdots \mathrm{C}$ interactions have a larger share of $27 \%$.

\section{Database survey}

A search of the Cambridge Crystallographic Database (WebCSD, November 2021; Groom et al., 2016) for 2-(4,5dihydrofuryl)silanes revealed solely the structures of tris(4,5dihydrofuran-2-yl)methylsilane and tris(4,5-dihydrofuran-2yl)phenylsilane published by our group previously (Krupp et al., 2020). A more extended search for 3-(4,5-dihydrofuryl)silane gave some structures with substituted dihydrofuran rings, such as [4-(4-fluorophenyl)-5-(4-nitrophenyl)-4,5-dihydrofuran-3-yl](trimethyl)silane (JIVLIM; Li \& Zhang, 2018), rac-5-phenyl-4-(t-butyldiphenylsilyl)-2,3-dihydrofuran2-carboxylic acid ethyl ester (PUXCAM; Evans et al., 2001) and $\quad\left(1^{\prime} S, 2 R\right)$-5-methyl-4-( $t$-butyldiphenylsilyl)-2,3-dihydrofuran-2-carboxylic acid (1'-phenylethyl)amide (PUXCEQ; Evans et al., 2001). Contrary to the here and previously presented 2-(4,5-dihydrofuryl)silanes (Krupp et al., 2020), the published 3-(4,5-dihydrofuryl)silanes do not show an elongated $\mathrm{Si}-\mathrm{C}(\mathrm{DHF})$ bond in comparison to the other substituents at the silicon atom. This can be attributed to the changed connection on the DHF ring. The slightly distorted tetrahedral silicon atom can be observed in all structures as well as the shortened $\mathrm{C}=\mathrm{C}$ double bond in the DHF ring.

\section{Synthesis and crystallization}

Bis(4,5-dihydrofuran-2-yl)dimethylsilane (1) as already described by Lukevics and co-workers (Lukevics et al., 1985) 
Table 4

Experimental details.

1

Crystal data

Chemical formula

$M_{\mathrm{r}}$

Crystal system, space group

Temperature $(\mathrm{K})$

$a, b, c(\AA)$

$\alpha, \beta, \gamma\left({ }^{\circ}\right)$

$V\left(\AA^{3}\right)$

Z

Radiation type

$\mu\left(\mathrm{mm}^{-1}\right)$

Crystal size (mm)

Data collection

Diffractometer

Absorption correction

$T_{\min }, T_{\max }$

No. of measured, independent and

observed $[I>2 \sigma(I)]$ reflections

$R_{\text {int }}$

$(\sin \theta / \lambda)_{\max }\left(\AA^{-1}\right)$

Refinement

$R\left[F^{2}>2 \sigma\left(F^{2}\right)\right], w R\left(F^{2}\right), S$

No. of reflections

No. of parameters

$\mathrm{H}$-atom treatment

$\Delta \rho_{\max }, \Delta \rho_{\min }\left(\mathrm{e} \AA^{-3}\right)$
$\mathrm{C}_{10} \mathrm{H}_{16} \mathrm{O}_{2} \mathrm{Si}$

196.32

Triclinic, $P \overline{1}$

100

8.2422 (3), 8.3075 (4), 8.2940 (4)

94.149 (2), 103.012 (1), 104.909 (1)

529.55 (4)

Mo $K \alpha$

0.19

$0.72 \times 0.66 \times 0.59$

Bruker D8 Venture

Multi-scan (SADABS; Krause et al., 2015)

$0.519,0.576$

256332, 11412, 10306

0.032

1.089

$0.026,0.089,1.06$

11412

182

$\mathrm{H}$ atoms treated by a mixture of independent

and constrained refinement.

$0.68,-0.32$
2

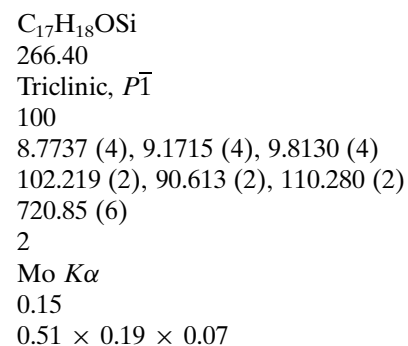

Bruker D8 Venture

Multi-scan (SADABS; Krause et al., 2015)

$0.713,0.747$

20409, 5826, 4937

0.030

0.787

$0.040,0.105,1.06$

5826

244

$\mathrm{H}$ atoms treated by a mixture of independent and constrained refinement.

$0.48,-0.27$

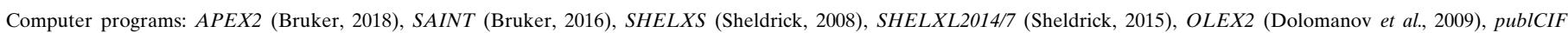
(Westrip, 2010) and Mercury (Macrae et al., 2020).

was synthesized by adding ${ }^{t} \mathrm{BuLi}(1.9 \mathrm{M}$ in pentane, $8.16 \mathrm{~mL}$, $15.5 \mathrm{mmol}, 2.0$ eq.) to a solution of 2,3-dihydrofuran $(1.09 \mathrm{~g}$, $15.5 \mathrm{mmol}, 2.0$ eq.) in diethyl ether at $243 \mathrm{~K}$ and subsequent stirring for an hour. Dichlorodimethylsilane (1.00 g, $7.75 \mathrm{mmol}, 1.0$ eq.) was added at $243 \mathrm{~K}$ and warmed to room temperature under stirring for $2 \mathrm{~h}$. All solids were filtered off inertly and all volatile components were removed in vacuo. After cleaning by Kugelrohr distillation (temperature: $373 \mathrm{~K}$, pressure: $\left.2.1 \times 10^{-1} \mathrm{mbar}\right)$, bis(4,5-dihydrofuran-2-yl)dimethylsilane (1) (1.50 g, $7.65 \mathrm{mmol}, 99 \%)$ was obtained as a colorless oil. By crystallization from diethyl ether at $193 \mathrm{~K}$, colorless blocks were obtained.

${ }^{1} \mathrm{H}$ NMR (400.25 MHz, $\left.\mathrm{C}_{6} \mathrm{D}_{6}\right): \delta=0.39\left[s, 6 \mathrm{H} ; \mathrm{Si}\left(\mathrm{CH}_{3}\right)_{2}\right]$, $2.27\left[d t,{ }^{3} J_{\mathrm{HH}}=2.57 \mathrm{~Hz},{ }^{3} J_{\mathrm{HH}}=9.78 \mathrm{~Hz}, 4 \mathrm{H} ; \mathrm{Si}\left(\mathrm{CCHCH}_{2}\right)_{2}\right]$, $4.07\left[t,{ }^{3} J_{\mathrm{HH}}=9.78 \mathrm{~Hz}, 4 \mathrm{H} ; \mathrm{Si}\left(\mathrm{COCH}_{2}\right)_{2}\right], 5.31\left[t,{ }^{3} J_{\mathrm{HH}}=\right.$ $\left.2.57 \mathrm{~Hz}, 2 \mathrm{H} ; \mathrm{Si}(\mathrm{CCH})_{2}\right] \mathrm{ppm}$.

$\left\{{ }^{1} \mathrm{H}\right\}{ }^{13} \mathrm{C}$ NMR $\left(100.6 \mathrm{MHz}, \mathrm{C}_{6} \mathrm{D}_{6}\right): \delta=-3.8$ [2C; $\left.\left(\mathrm{SiCH}_{3}\right)_{2}\right]$, 31.6 [2C; $\left.\mathrm{Si}\left(\mathrm{CCHCH}_{2}\right)_{2}\right)$ ], 71.8 [2C; $\left.\mathrm{Si}\left(\mathrm{COCH}_{2}\right)_{2}\right], 113.4$ [2C; $\left.\mathrm{Si}\left(\mathrm{COCH}_{2}\right)_{2}\right], 160.0$ [2C; $\left.\mathrm{Si}(\mathrm{CO})_{2}\right] \mathrm{ppm}$.

$\left\{{ }^{1} \mathrm{H}\right\}^{29} \mathrm{Si}$ NMR $\left(79.52 \mathrm{MHz}, \quad \mathrm{C}_{6} \mathrm{D}_{6}\right): \delta=-22.29 \quad[1 \mathrm{Si}$; $\left.\mathrm{Si}(\mathrm{DHF})_{2}\right] \mathrm{ppm}$.

GC/EI-MS: $t_{\mathrm{R}}=3.94 \mathrm{~min}\left[353 \mathrm{~K}(1 \mathrm{~min})-40 \mathrm{~K} \mathrm{~min}^{-1}-\right.$ $543 \mathrm{~K}(5.5 \mathrm{~min})] ; \mathrm{m} / z(\%): 196(94)\left[M^{+}\right], 181(2)\left[(M-\mathrm{Me})^{+}\right]$, $167(14)\left[(M-\mathrm{CHO})^{+}\right], 153(40)\left[\left(M-\mathrm{C}_{2} \mathrm{H}_{3} \mathrm{O}\right)^{+}\right], 97(100)$ $\left[(\mathrm{SiDHF})^{+}\right]$.

(4,5-Dihydrofuran-2-yl)(methyl)diphenylsilane (2), already described by Tsai and co-workers (Tsai et al., 1992) by cyclization of a haloacylsilane, was synthesized analogously to 1. ${ }^{t} \mathrm{BuLi}$ (1.90 $\mathrm{M}$ in pentane, $21.5 \mathrm{mmol}, 11.3 \mathrm{~mL}, 1.00$ eq.) was slowly added dropwise to a solution of 2,3-dihydrofuran (1.51 g, $21.5 \mathrm{mmol}, 1.00$ eq.) in diethyl ether $(80 \mathrm{~mL})$ at $243 \mathrm{~K}$ and the reaction solution was stirred for $1 \mathrm{~h}$ at this temperature. Methyldiphenylchlorosilane (5.00 g, $21.5 \mathrm{mmol}, 1.00 \mathrm{eq}$.) was then added at $243 \mathrm{~K}$ and the reaction solution was stirred at room temperature overnight. All solids were separated by inert filtration and the solvent was removed in vacuo. The residue was purified by Kugelrohr distillation (temperature: $453 \mathrm{~K}$, pressure: $\left.2.0 \times 10^{-1} \mathrm{mbar}\right)$ and the product $2(5.11 \mathrm{~g}$, $19.2 \mathrm{mmol}, 89 \%$ ) was obtained as a colorless liquid. By crystallization from pentane at $193 \mathrm{~K}$, colorless platelets were obtained.

${ }^{1} \mathrm{H}$ NMR (400.25 MHz, $\left.\mathrm{C}_{6} \mathrm{D}_{6}\right): \delta=0.69\left(s, 3 \mathrm{H} ; \mathrm{SiCH}_{3}\right), 2.24$ $\left(d t,{ }^{3} J_{\mathrm{HH}}=2.57 \mathrm{~Hz},{ }^{3} J_{\mathrm{HH}}=9.66 \mathrm{~Hz}, 2 \mathrm{H} ; \mathrm{SiCCHCH}_{2}\right), 4.07(t$, $\left.{ }^{3} J_{\mathrm{HH}}=9.66 \mathrm{~Hz}, 2 \mathrm{H} ; \mathrm{SiCOCH}_{2}\right), 5.20\left(t,{ }^{3} J_{\mathrm{HH}}=2.57 \mathrm{~Hz}, 1 \mathrm{H}\right.$; $\mathrm{SiCCH}), 7.18-7.21$ ( $\left.m, 6 \mathrm{H} ; \mathrm{CH}_{\text {ortho,para }}\right), 7.71-7.73(\mathrm{~m}, 4 \mathrm{H}$; $\left.\mathrm{CH}_{\text {meta }}\right) \mathrm{ppm}$.

$\left\{{ }^{1} \mathrm{H}\right\}{ }^{13} \mathrm{C}$ NMR (100.65 MHz, $\left.\mathrm{C}_{6} \mathrm{D}_{6}\right): \delta=-3.9\left(1 \mathrm{C} ; \mathrm{SiCH}_{3}\right)$, $31.3\left(1 \mathrm{C} ; \mathrm{SiCCHCH}_{2}\right), 71.1\left(1 \mathrm{C} ; \mathrm{SiCOCH}_{2}\right), 115.5(1 \mathrm{C}$; $\mathrm{SiCCH}), 128.5$ (2C, $\left.C_{\text {ortho }}\right), 130.2$ (1C, $\left.C_{\text {para }}\right), 135.8$ (2C, $\left.C_{\text {meta }}\right)$, $135.8\left(1 \mathrm{C}, C_{\text {ipso }}\right), 160.2(1 \mathrm{C}, \mathrm{SiCO}) \mathrm{ppm}$.

$\left\{{ }^{1} \mathrm{H}\right\}{ }^{29} \mathrm{Si}$ NMR $\left(79.52 \mathrm{MHz}, \mathrm{C}_{6} \mathrm{D}_{6}\right): \delta=-19.51(s, 1 \mathrm{Si}$; SiDHF) ppm.

GC/EI-MS: $t_{\mathrm{R}}=5.97 \mathrm{~min}\left[353 \mathrm{~K}(1 \mathrm{~min})-40 \mathrm{~K} \mathrm{~min}^{-1}-\right.$ $543 \mathrm{~K} \quad(5.5 \mathrm{~min})] ; \quad \mathrm{m} / z \quad(\%): 266(100) \quad\left[M^{+}\right], \quad 251(33)$ 
$\left[\begin{array}{llll}(M-\mathrm{Me})^{+}\end{array}\right], 238(27) \quad\left[\left(M-\mathrm{C}_{2} \mathrm{H}_{4}\right)^{+}\right], \quad 222(20)$ $\left[\left(M-\mathrm{C}_{2} \mathrm{H}_{4} \mathrm{O}\right)^{+}\right], 197(75)\left[(M-\mathrm{DHF})^{+}\right], 105(52)\left[(\mathrm{SiPh})^{+}\right]$, $77(6)\left[(\mathrm{Ph})^{+}\right]$.

\section{Refinement}

Crystal data, data collection and structure refinement details are summarized in Table 4 . $\mathrm{H}$ atoms were positioned geometrically $(\mathrm{C}-\mathrm{H}=0.95-1.00 \AA)$ and were refined using a riding model, with $U_{\text {iso }}(\mathrm{H})=1.2 U_{\text {eq }}(\mathrm{C})$ for $\mathrm{CH}_{2}$ and $\mathrm{CH}$ hydrogen atoms and $U_{\text {iso }}(\mathrm{H})=1.5 U_{\text {eq }}(\mathrm{C})$ for $\mathrm{CH}_{3}$ hydrogen atoms. Hydrogen atoms $\mathrm{H} 2$ and $\mathrm{H} 6$ for compound $\mathbf{1}$ and H2, H15 and $\mathrm{H} 17 \mathrm{C}$ for compound $\mathbf{2}$ were refined independently.

\section{Funding information}

Funding for this research was provided by: Fonds der Chemischen Industrie (scholarship to ERB).

\section{References}

Allen, F. H., Kennard, O., Watson, D. G., Brammer, L., Orpen, A. G. \& Taylor, R. (1987). J. Chem. Soc. Perkin Trans. 2, 12, 1-19.

Bruker (2016). SAINT. Bruker AXS Inc., Madison, Wisconsin, USA. Bruker (2018). APEX2. Bruker AXS Inc., Madison, Wisconsin, USA.

Colquhoun, V. P., Abele, B. C. \& Strohmann, C. (2011). Organometallics, 30, 5408-5414.

Colquhoun, V. P. \& Strohmann, C. (2012). Dalton Trans. 41, 18971902.

Desiraju, G. \& Steiner, T. (2001). The Weak Hydrogen Bond. Oxford University Press.

Dolomanov, O. V., Bourhis, L. J., Gildea, R. J., Howard, J. A. K. \& Puschmann, H. (2009). J. Appl. Cryst. 42, 339-341.

Etter, M. C., MacDonald, J. C. \& Bernstein, J. (1990). Acta Cryst. B46, 256-262.
Evans, D. A., Sweeney, Z. K., Rovis, T. \& Tedrow, J. S. (2001). J. Am. Chem. Soc. 123, 12095-12096.

Gevorgyan, V., Borisova, L. \& Lukevics, E. (1989). J. Organomet. Chem. 368, 19-21.

Gevorgyan, V., Borisova, L. \& Lukevics, E. (1990). J. Organomet. Chem. 393, 57-67.

Gevorgyan, V., Borisova, L. \& Lukevics, E. (1992). J. Organomet. Chem. 441, 381-387.

Gevorgyan, V., Borisova, L. \& Lukevics, E. (1997). J. Organomet. Chem. 527, 295-296.

Groom, C. R., Bruno, I. J., Lightfoot, M. P. \& Ward, S. C. (2016). Acta Cryst. B72, 171-179.

Krause, L., Herbst-Irmer, R., Sheldrick, G. M. \& Stalke, D. (2015). J. Appl. Cryst. 48, 3-10.

Krupp, A., Barth, E. R., Seymen, R. \& Strohmann, C. (2020). Acta Cryst. E76, 1514-1519.

Labrecque, D., Nwe, K. T. \& Chan, T. H. (1994). Organometallics, 13, 332-335.

Li, T. \& Zhang, L. (2018). J. Am. Chem. Soc. 140, 17439-17443.

Lukevics, E., Gevorgyan, V. \& Borisova, L. (1997). Chem. Heterocycl. Compd. 33, 161-163.

Lukevics, E., Gevorgyan, V. N., Goldberg, Y. S. \& Shymanska, M. V. (1985). J. Organomet. Chem. 294, 163-171.

Macrae, C. F., Sovago, I., Cottrell, S. J., Galek, P. T. A., McCabe, P., Pidcock, E., Platings, M., Shields, G. P., Stevens, J. S., Towler, M. \& Wood, P. A. (2020). J. Appl. Cryst. 53, 226-235.

McKinnon, J. J., Jayatilaka, D. \& Spackman, M. A. (2007). Chem. Commun. pp. 3814-3816.

Sheldrick, G. M. (2008). Acta Cryst. A64, 112-122.

Sheldrick, G. M. (2015). Acta Cryst. C71, 3-8.

Spackman, M. A. \& Jayatilaka, D. (2009). CrystEngComm, 11, 19-32.

Spackman, P. R., Turner, M. J., McKinnon, J. J., Wolff, S. K., Grimwood, D. J., Jayatilaka, D. \& Spackman, M. A. (2021). J. Appl. Cryst. 54, 1006-1011.

Tsai, Y.-M., Nieh, H.-C. \& Cherng, C.-D. (1992). J. Org. Chem. 57, 7010-7012.

Westrip, S. P. (2010). J. Appl. Cryst. 43, 920-925. 


\section{supporting information}

Acta Cryst. (2022). E78, 23-28 [https://doi.org/10.1107/S2056989021012548]

Crystal structures and Hirshfeld surface analyses of bis(4,5-dihydrofuran-2yl)dimethylsilane and (4,5-dihydrofuran-2-yl)(methyl)diphenylsilane

\section{Annika Schmidt, Anna Krupp, Eva Rebecca Barth and Carsten Strohmann}

Computing details

For both structures, data collection: APEX2 (Bruker, 2018); cell refinement: SAINT (Bruker, 2016); data reduction: SAINT (Bruker, 2016); program(s) used to solve structure: SHELXS (Sheldrick, 2008); program(s) used to refine structure: SHELXL2014/7 (Sheldrick, 2015); molecular graphics: OLEX2 (Dolomanov et al., 2009); software used to prepare material for publication: OLEX2 (Dolomanov et al., 2009), CrystalExplorer21 (Spackman et al., 2021), publCIF (Westrip, 2010), Mercury (Macrae et al., 2020).

Bis(4,5-dihydrofuran-2-yl)dimethylsilane (1)

Crystal data

$\mathrm{C}_{10} \mathrm{H}_{16} \mathrm{O}_{2} \mathrm{Si}$

$M_{r}=196.32$

Triclinic, $P \overline{1}$

$a=8.2422(3) \AA$

$b=8.3075(4) \AA$

$c=8.2940(4) \AA$

$\alpha=94.149(2)^{\circ}$

$\beta=103.012(1)^{\circ}$

$\gamma=104.909(1)^{\circ}$

$V=529.55(4) \AA^{3}$

\section{Data collection}

Bruker D8 Venture diffractometer

Radiation source: microfocus sealed X-ray tube, Incoatec $\mathrm{I} \mu \mathrm{s}$

Mirror optics monochromator

Detector resolution: 7.9 pixels $\mathrm{mm}^{-1}$

$\omega$ and $\varphi$ scans

Absorption correction: multi-scan

(SADABS; Krause et al., 2015)

\section{Refinement}

Refinement on $F^{2}$

Least-squares matrix: full $R\left[F^{2}>2 \sigma\left(F^{2}\right)\right]=$ 0.026

$w R\left(F^{2}\right)=0.089$

$S=1.06$
$Z=2$

$F(000)=212$

$D_{\mathrm{x}}=1.231 \mathrm{Mg} \mathrm{m}^{-3}$

Mo $K \alpha$ radiation, $\lambda=0.71073 \AA$

Cell parameters from 8689 reflections

$\theta=2.5-20.9^{\circ}$

$\mu=0.19 \mathrm{~mm}^{-1}$

$T=100 \mathrm{~K}$

Block, colourless

$0.72 \times 0.66 \times 0.59 \mathrm{~mm}$

$T_{\min }=0.519, T_{\max }=0.576$

256332 measured reflections

11412 independent reflections

10306 reflections with $I>2 \sigma(I)$

$R_{\text {int }}=0.032$

$\theta_{\text {max }}=50.7^{\circ}, \theta_{\min }=2.6^{\circ}$

$h=-16 \rightarrow 17$

$k=-18 \rightarrow 18$

$l=-18 \rightarrow 18$

11412 reflections

182 parameters

0 restraints

Primary atom site location: iterative

Hydrogen site location: difference Fourier map

H_atoms_treated_by_a_mixture_of_independent_and_constrained_refinem 
$w=1 /\left[\sigma^{2}\left(F_{\mathrm{o}}^{2}\right)+(0.0535 P)^{2}+0.0207 P\right]$

where $P=\left(F_{\mathrm{o}}^{2}+2 F_{\mathrm{c}}{ }^{2}\right) / 3$

$(\Delta / \sigma)_{\max }=0.002$

$$
\Delta \rho_{\max }=0.68 \text { e } \AA^{-3}
$$

\section{Special details}

Geometry. All esds (except the esd in the dihedral angle between two 1.s. planes) are estimated using the full covariance matrix. The cell esds are taken into account individually in the estimation of esds in distances, angles and torsion angles; correlations between esds in cell parameters are only used when they are defined by crystal symmetry. An approximate (isotropic) treatment of cell esds is used for estimating esds involving 1.s. planes.

Fractional atomic coordinates and isotropic or equivalent isotropic displacement parameters $\left(\AA^{2}\right)$

\begin{tabular}{|c|c|c|c|c|}
\hline & $x$ & $y$ & $z$ & $U_{\text {iso }} * / U_{\text {eq }}$ \\
\hline Si1 & $0.64185(2)$ & $0.82439(2)$ & $0.20897(2)$ & $0.01354(2)$ \\
\hline $\mathrm{O} 1$ & $0.28272(3)$ & $0.68623(3)$ & $0.09023(4)$ & $0.02227(4)$ \\
\hline $\mathrm{O} 2$ & $0.70145(4)$ & $0.71875(3)$ & $0.52219(3)$ & $0.02213(4)$ \\
\hline $\mathrm{C} 1$ & $0.41042(3)$ & $0.81964(3)$ & $0.19462(3)$ & $0.01504(3)$ \\
\hline $\mathrm{C} 2$ & $0.34535(4)$ & $0.92843(4)$ & $0.26763(4)$ & $0.01740(4)$ \\
\hline $\mathrm{C} 3$ & $0.14995(4)$ & $0.87390(4)$ & $0.21112(4)$ & $0.02057(4)$ \\
\hline $\mathrm{H} 3 \mathrm{~A}$ & $0.0906(12)$ & $0.8525(12)$ & $0.3024(12)$ & $0.033(2)^{*}$ \\
\hline $\mathrm{H} 3 \mathrm{~B}$ & $0.1063(12)$ & 0.9549 (11) & $0.1543(11)$ & $0.032(2)^{*}$ \\
\hline $\mathrm{C} 4$ & $0.11591(4)$ & $0.70688(5)$ & $0.10008(5)$ & $0.02242(5)$ \\
\hline $\mathrm{H} 4 \mathrm{~A}$ & $0.0538(11)$ & $0.6100(11)$ & $0.1453(11)$ & $0.032(2)^{*}$ \\
\hline $\mathrm{H} 4 \mathrm{~B}$ & $0.0486(12)$ & $0.6963(12)$ & $-0.0129(12)$ & $0.037(2)^{*}$ \\
\hline $\mathrm{C} 5$ & $0.70144(3)$ & $0.67768(3)$ & $0.35736(3)$ & $0.01419(3)$ \\
\hline C6 & $0.73403(4)$ & $0.52967(3)$ & $0.32841(3)$ & $0.01609(4)$ \\
\hline $\mathrm{C} 7$ & $0.75301(4)$ & $0.44890(4)$ & $0.48628(4)$ & $0.01889(4)$ \\
\hline $\mathrm{H} 7 \mathrm{~A}$ & $0.8533(11)$ & $0.4108(11)$ & $0.5151(11)$ & $0.0284(18)^{*}$ \\
\hline H7B & $0.6498(10)$ & $0.3515(11)$ & $0.4771(10)$ & $0.0251(17)^{*}$ \\
\hline $\mathrm{C} 8$ & $0.75865(6)$ & $0.59310(5)$ & $0.61494(4)$ & $0.02397(6)$ \\
\hline H8A & $0.6831(12)$ & $0.5602(11)$ & $0.6876(12)$ & $0.033(2)^{*}$ \\
\hline $\mathrm{H} 8 \mathrm{~B}$ & $0.8762(14)$ & $0.6488(14)$ & $0.6853(14)$ & $0.048(3)^{*}$ \\
\hline C9 & $0.77560(4)$ & $1.04490(4)$ & $0.28985(5)$ & $0.02162(5)$ \\
\hline H9A & $0.7566(12)$ & $1.0847(12)$ & $0.3964(12)$ & $0.037(2)^{*}$ \\
\hline H9B & $0.9008(14)$ & $1.0571(13)$ & $0.3185(14)$ & $0.048(3)^{*}$ \\
\hline $\mathrm{H} 9 \mathrm{C}$ & $0.7555(14)$ & $1.1226(14)$ & $0.2124(13)$ & $0.047(3)^{*}$ \\
\hline $\mathrm{C} 10$ & $0.66177(4)$ & $0.74698(4)$ & $0.00048(4)$ & $0.01893(4)$ \\
\hline $\mathrm{H} 10 \mathrm{~A}$ & $0.6108(13)$ & $0.8064(13)$ & $-0.0859(13)$ & $0.040(2)^{*}$ \\
\hline H10B & $0.6006(14)$ & $0.6296(14)$ & $-0.0355(13)$ & $0.045(3)^{*}$ \\
\hline $\mathrm{H} 10 \mathrm{C}$ & $0.7765(13)$ & $0.7669(12)$ & $-0.0041(12)$ & $0.036(2)^{*}$ \\
\hline H6 & $0.7325(10)$ & $0.4798(10)$ & $0.2272(10)$ & $0.0225(16)^{*}$ \\
\hline $\mathrm{H} 2$ & $0.4137(12)$ & $1.0273(11)$ & $0.3403(11)$ & $0.033(2)^{*}$ \\
\hline
\end{tabular}

Atomic displacement parameters $\left(\AA^{2}\right)$

\begin{tabular}{lllllll}
\hline & $U^{11}$ & $U^{22}$ & $U^{33}$ & $U^{12}$ & $U^{13}$ & $U^{23}$ \\
\hline Si1 & $0.01424(3)$ & $0.01281(3)$ & $0.01491(3)$ & $0.00527(2)$ & $0.00450(2)$ & $0.00244(2)$ \\
O1 & $0.01651(7)$ & $0.02070(9)$ & $0.02720(10)$ & $0.00574(6)$ & $0.00287(7)$ & $-0.00545(7)$ \\
O2 & $0.03579(12)$ & $0.02135(9)$ & $0.01466(7)$ & $0.01572(9)$ & $0.00835(7)$ & $0.00268(6)$
\end{tabular}


supporting information

\begin{tabular}{lllllll} 
C1 & $0.01497(7)$ & $0.01501(7)$ & $0.01637(8)$ & $0.00611(6)$ & $0.00417(6)$ & $0.00253(6)$ \\
C2 & $0.01676(8)$ & $0.01709(8)$ & $0.01982(9)$ & $0.00723(7)$ & $0.00523(7)$ & $0.00100(7)$ \\
C3 & $0.01692(9)$ & $0.02220(11)$ & $0.02551(12)$ & $0.00930(8)$ & $0.00669(8)$ & $0.00381(9)$ \\
C4 & $0.01543(9)$ & $0.02642(13)$ & $0.02320(11)$ & $0.00544(8)$ & $0.00243(8)$ & $-0.00117(9)$ \\
C5 & $0.01579(7)$ & $0.01407(7)$ & $0.01381(7)$ & $0.00590(6)$ & $0.00406(6)$ & $0.00171(5)$ \\
C6 & $0.01916(9)$ & $0.01436(8)$ & $0.01617(8)$ & $0.00689(6)$ & $0.00502(7)$ & $0.00142(6)$ \\
C7 & $0.02225(10)$ & $0.01595(8)$ & $0.02068(10)$ & $0.00752(7)$ & $0.00644(8)$ & $0.00561(7)$ \\
C8 & $0.03680(17)$ & $0.02108(11)$ & $0.01494(9)$ & $0.01061(11)$ & $0.00498(9)$ & $0.00432(8)$ \\
C9 & $0.02043(10)$ & $0.01447(9)$ & $0.02932(13)$ & $0.00399(7)$ & $0.00669(9)$ & $0.00107(8)$ \\
C10 & $0.02063(10)$ & $0.02226(10)$ & $0.01611(9)$ & $0.00769(8)$ & $0.00686(7)$ & $0.00360(7)$ \\
\hline
\end{tabular}

Geometric parameters $\left(\hat{A},{ }^{\circ}\right)$

\begin{tabular}{|c|c|c|c|}
\hline $\mathrm{Si} 1-\mathrm{C} 1$ & $1.8742(3)$ & $\mathrm{C} 4-\mathrm{H} 4 \mathrm{~B}$ & $0.961(10)$ \\
\hline $\mathrm{Si} 1-\mathrm{C} 5$ & $1.8693(3)$ & $\mathrm{C} 5-\mathrm{C} 6$ & 1.3409 (4) \\
\hline Si1-C9 & $1.8631(3)$ & $\mathrm{C} 6-\mathrm{C} 7$ & $1.5093(4)$ \\
\hline $\mathrm{Si} 1-\mathrm{C} 10$ & $1.8579(3)$ & $\mathrm{C} 6-\mathrm{H} 6$ & $0.904(8)$ \\
\hline $\mathrm{O} 1-\mathrm{C} 1$ & 1.3915 (4) & $\mathrm{C} 7-\mathrm{H} 7 \mathrm{~A}$ & $0.947(9)$ \\
\hline $\mathrm{O} 1-\mathrm{C} 4$ & 1.4479 (4) & C7-H7B & $0.996(8)$ \\
\hline $\mathrm{O} 2-\mathrm{C} 5$ & $1.3849(3)$ & $\mathrm{C} 7-\mathrm{C} 8$ & $1.5298(5)$ \\
\hline $\mathrm{O} 2-\mathrm{C} 8$ & $1.4525(4)$ & $\mathrm{C} 8-\mathrm{H} 8 \mathrm{~A}$ & $0.965(9)$ \\
\hline $\mathrm{C} 1-\mathrm{C} 2$ & $1.3370(4)$ & $\mathrm{C} 8-\mathrm{H} 8 \mathrm{~B}$ & $0.984(11)$ \\
\hline $\mathrm{C} 2-\mathrm{C} 3$ & $1.5075(4)$ & C9-H9A & $0.980(10)$ \\
\hline $\mathrm{C} 2-\mathrm{H} 2$ & $0.945(9)$ & C9-H9B & $0.982(11)$ \\
\hline $\mathrm{C} 3-\mathrm{H} 3 \mathrm{~A}$ & $0.992(9)$ & $\mathrm{C} 9-\mathrm{H} 9 \mathrm{C}$ & $0.961(11)$ \\
\hline $\mathrm{C} 3-\mathrm{H} 3 \mathrm{~B}$ & $0.948(9)$ & $\mathrm{C} 10-\mathrm{H} 10 \mathrm{~A}$ & $0.978(10)$ \\
\hline $\mathrm{C} 3-\mathrm{C} 4$ & $1.5331(5)$ & $\mathrm{C} 10-\mathrm{H} 10 \mathrm{~B}$ & $0.965(11)$ \\
\hline $\mathrm{C} 4-\mathrm{H} 4 \mathrm{~A}$ & $0.984(9)$ & $\mathrm{C} 10-\mathrm{H} 10 \mathrm{C}$ & $0.927(10)$ \\
\hline $\mathrm{C} 5-\mathrm{Si} 1-\mathrm{C} 1$ & $108.189(12)$ & $\mathrm{C} 6-\mathrm{C} 5-\mathrm{O} 2$ & $113.03(2)$ \\
\hline $\mathrm{C} 9-\mathrm{Si} 1-\mathrm{C} 1$ & $106.801(14)$ & $\mathrm{C} 5-\mathrm{C} 6-\mathrm{C} 7$ & $109.72(2)$ \\
\hline $\mathrm{C} 9-\mathrm{Si} 1-\mathrm{C} 5$ & $110.770(15)$ & $\mathrm{C} 5-\mathrm{C} 6-\mathrm{H} 6$ & $125.3(5)$ \\
\hline $\mathrm{C} 10-\mathrm{Si} 1-\mathrm{C} 1$ & $109.191(13)$ & $\mathrm{C} 7-\mathrm{C} 6-\mathrm{H} 6$ & $124.7(5)$ \\
\hline $\mathrm{C} 10-\mathrm{Si} 1-\mathrm{C} 5$ & $108.128(13)$ & $\mathrm{C} 6-\mathrm{C} 7-\mathrm{H} 7 \mathrm{~A}$ & $114.8(5)$ \\
\hline $\mathrm{C} 10-\mathrm{Si} 1-\mathrm{C} 9$ & $113.628(16)$ & $\mathrm{C} 6-\mathrm{C} 7-\mathrm{H} 7 \mathrm{~B}$ & $110.3(5)$ \\
\hline $\mathrm{C} 1-\mathrm{O} 1-\mathrm{C} 4$ & $107.60(2)$ & $\mathrm{C} 6-\mathrm{C} 7-\mathrm{C} 8$ & $101.18(2)$ \\
\hline $\mathrm{C} 5-\mathrm{O} 2-\mathrm{C} 8$ & $107.15(2)$ & $\mathrm{H} 7 \mathrm{~A}-\mathrm{C} 7-\mathrm{H} 7 \mathrm{~B}$ & $107.9(7)$ \\
\hline $\mathrm{O} 1-\mathrm{C} 1-\mathrm{Si} 1$ & $117.093(19)$ & $\mathrm{C} 8-\mathrm{C} 7-\mathrm{H} 7 \mathrm{~A}$ & $111.6(5)$ \\
\hline $\mathrm{C} 2-\mathrm{C} 1-\mathrm{Si} 1$ & $129.94(2)$ & $\mathrm{C} 8-\mathrm{C} 7-\mathrm{H} 7 \mathrm{~B}$ & $110.9(5)$ \\
\hline $\mathrm{C} 2-\mathrm{C} 1-\mathrm{O} 1$ & $112.95(2)$ & $\mathrm{O} 2-\mathrm{C} 8-\mathrm{C} 7$ & $106.95(2)$ \\
\hline $\mathrm{C} 1-\mathrm{C} 2-\mathrm{C} 3$ & $110.20(3)$ & $\mathrm{O} 2-\mathrm{C} 8-\mathrm{H} 8 \mathrm{~A}$ & $107.7(6)$ \\
\hline $\mathrm{C} 1-\mathrm{C} 2-\mathrm{H} 2$ & $124.1(5)$ & $\mathrm{O} 2-\mathrm{C} 8-\mathrm{H} 8 \mathrm{~B}$ & $106.9(6)$ \\
\hline $\mathrm{C} 3-\mathrm{C} 2-\mathrm{H} 2$ & $125.7(5)$ & $\mathrm{C} 7-\mathrm{C} 8-\mathrm{H} 8 \mathrm{~A}$ & $113.7(6)$ \\
\hline $\mathrm{C} 2-\mathrm{C} 3-\mathrm{H} 3 \mathrm{~A}$ & $114.7(5)$ & $\mathrm{C} 7-\mathrm{C} 8-\mathrm{H} 8 \mathrm{~B}$ & $113.2(6)$ \\
\hline $\mathrm{C} 2-\mathrm{C} 3-\mathrm{H} 3 \mathrm{~B}$ & $111.8(5)$ & $\mathrm{H} 8 \mathrm{~A}-\mathrm{C} 8-\mathrm{H} 8 \mathrm{~B}$ & $108.0(8)$ \\
\hline $\mathrm{C} 2-\mathrm{C} 3-\mathrm{C} 4$ & $101.57(2)$ & Si1—C9—H9A & $111.7(5)$ \\
\hline $\mathrm{H} 3 \mathrm{~A}-\mathrm{C} 3-\mathrm{H} 3 \mathrm{~B}$ & $106.4(7)$ & Si1-C9-H9B & $113.1(6)$ \\
\hline $\mathrm{C} 4-\mathrm{C} 3-\mathrm{H} 3 \mathrm{~A}$ & $108.8(5)$ & $\mathrm{Si} 1-\mathrm{C} 9-\mathrm{H} 9 \mathrm{C}$ & $113.1(6)$ \\
\hline
\end{tabular}




$\begin{array}{llll}\mathrm{C} 4-\mathrm{C} 3-\mathrm{H} 3 \mathrm{~B} & 113.8(5) & \mathrm{H} 9 \mathrm{~A}-\mathrm{C} 9-\mathrm{H} 9 \mathrm{~B} & 102.7(8) \\ \mathrm{O} 1-\mathrm{C} 4-\mathrm{C} 3 & 107.47(3) & \mathrm{H} 9 \mathrm{~A}-\mathrm{C} 9-\mathrm{H} 9 \mathrm{C} & 108.8(8) \\ \mathrm{O} 1-\mathrm{C} 4-\mathrm{H} 4 \mathrm{~A} & 108.9(5) & \mathrm{H} 9 \mathrm{~B}-\mathrm{C} 9-\mathrm{H} 9 \mathrm{C} & 106.8(9) \\ \mathrm{O} 1-\mathrm{C} 4-\mathrm{H} 4 \mathrm{~B} & 106.6(6) & \mathrm{Si}-\mathrm{C} 10-\mathrm{H} 10 \mathrm{~A} & 111.1(6) \\ \mathrm{C} 3-\mathrm{C} 4-\mathrm{H} 4 \mathrm{~A} & 112.4(5) & \mathrm{Si} 1-\mathrm{C} 10-\mathrm{H} 10 \mathrm{~B} & 112.9(6) \\ \mathrm{C} 3-\mathrm{C} 4-\mathrm{H} 4 \mathrm{~B} & 116.7(6) & \mathrm{Si} 1-\mathrm{C} 10-\mathrm{H} 10 \mathrm{C} & 112.3(6) \\ \mathrm{H} 4 \mathrm{~A}-\mathrm{C} 4-\mathrm{H} 4 \mathrm{~B} & 104.5(8) & \mathrm{H} 10 \mathrm{~A}-\mathrm{C} 10-\mathrm{H} 10 \mathrm{~B} & 105.1(8) \\ \mathrm{O} 2-\mathrm{C} 5-\mathrm{Si} & 116.686(18) & \mathrm{H} 10 \mathrm{~A}-\mathrm{C} 10-\mathrm{H} 10 \mathrm{C} & 104.8(8) \\ \mathrm{C} 6-\mathrm{C} 5-\mathrm{Si} 1 & 130.12(2) & \mathrm{H} 10 \mathrm{~B}-\mathrm{C} 10-\mathrm{H} 10 \mathrm{C} & 110.1(8) \\ & & & \\ \mathrm{Si} 1-\mathrm{C} 1-\mathrm{C} 2-\mathrm{C} 3 & 177.42(2) & \mathrm{C} 5-\mathrm{O} 2-\mathrm{C} 8-\mathrm{C} 7 & -13.01(4) \\ \mathrm{Si} 1-\mathrm{C} 5-\mathrm{C} 6-\mathrm{C} 7 & -172.51(2) & \mathrm{C} 5-\mathrm{C} 6-\mathrm{C} 7-\mathrm{C} 8 & -10.06(3) \\ \mathrm{O} 1-\mathrm{C} 1-\mathrm{C} 2-\mathrm{C} 3 & -1.01(4) & \mathrm{C} 6-\mathrm{C} 7-\mathrm{C} 8-\mathrm{O} 2 & 13.68(4) \\ \mathrm{O} 2-\mathrm{C} 5-\mathrm{C} 6-\mathrm{C} 7 & 2.56(4) & \mathrm{C} 8-\mathrm{O} 2-\mathrm{C} 5-\mathrm{Si} 1 & -177.44(2) \\ \mathrm{C} 1-\mathrm{Si} 1-\mathrm{C} 5-\mathrm{O} 2 & -64.48(2) & \mathrm{C} 8-\mathrm{O} 2-\mathrm{C} 5-\mathrm{C} 6 & 6.78(4) \\ \mathrm{C} 1-\mathrm{Si} 1-\mathrm{C} 5-\mathrm{C} 6 & 110.44(3) & \mathrm{C} 9-\mathrm{Si} 1-\mathrm{C} 1-\mathrm{O} 1 & 160.08(2) \\ \mathrm{C} 1-\mathrm{O} 1-\mathrm{C} 4-\mathrm{C} 3 & 4.15(4) & \mathrm{C} 9-\mathrm{Si}-\mathrm{C} 1-\mathrm{C} 2 & -18.30(3) \\ \mathrm{C} 1-\mathrm{C} 2-\mathrm{C} 3-\mathrm{C} 4 & 3.38(4) & \mathrm{C} 9-\mathrm{Si} 1-\mathrm{C} 5-\mathrm{O} 2 & 52.26(3) \\ \mathrm{C} 2-\mathrm{C} 3-\mathrm{C} 4-\mathrm{O} 1 & -4.46(4) & \mathrm{C} 9-\mathrm{Si} 1-\mathrm{C} 5-\mathrm{C} 6 & -132.81(3) \\ \mathrm{C} 4-\mathrm{O} 1-\mathrm{C} 1-\mathrm{Si} 1 & 179.30(2) & \mathrm{C} 10-\mathrm{Si}-\mathrm{C} 1-\mathrm{O} 1 & 36.82(3) \\ \mathrm{C} 4-\mathrm{O} 1-\mathrm{C} 1-\mathrm{C} 2 & -2.04(4) & \mathrm{C} 10-\mathrm{Si} 1-\mathrm{C} 1-\mathrm{C} 2 & -141.56(3) \\ \mathrm{C} 5-\mathrm{Si1}-\mathrm{C} 1-\mathrm{O} 1 & -80.64(2) & \mathrm{C} 10-\mathrm{Si} 1-\mathrm{C} 5-\mathrm{O} 2 & 177.38(2) \\ \mathrm{C} 5-\mathrm{Si} 1-\mathrm{C} 1-\mathrm{C} 2 & 100.98(3) & \mathrm{C} 10-\mathrm{Si} 1-\mathrm{C} 5-\mathrm{C} 6 & -7.70(3) \\ & & & \end{array}$

(4,5-Dihydrofuran-2-yl)(methyl)diphenylsilane (2)

\section{Crystal data}

$\mathrm{C}_{17} \mathrm{H}_{18} \mathrm{OSi}$

$M_{r}=266.40$

Triclinic, $P \overline{1}$

$a=8.7737$ (4) $\AA$

$b=9.1715(4) \AA$

$c=9.8130(4) \AA$

$\alpha=102.219(2)^{\circ}$

$\beta=90.613(2)^{\circ}$

$\gamma=110.280(2)^{\circ}$

$V=720.85(6) \AA^{3}$

\section{Data collection}

Bruker D8 Venture diffractometer

Radiation source: microfocus sealed X-ray tube, INCOATEC microfocus sealed tube, Iys 3.0

Multilayer optics monochromator

Detector resolution: 10.4167 pixels $\mathrm{mm}^{-1}$

$\varphi$ and $\omega$ scans

Absorption correction: multi-scan

(SADABS; Krause et al., 2015)
$Z=2$

$F(000)=284$

$D_{\mathrm{x}}=1.227 \mathrm{Mg} \mathrm{m}^{-3}$

Mo $K \alpha$ radiation, $\lambda=0.71073 \AA$

Cell parameters from 8775 reflections

$\theta=2.5-36.3^{\circ}$

$\mu=0.15 \mathrm{~mm}^{-1}$

$T=100 \mathrm{~K}$

Plate, colourless

$0.51 \times 0.19 \times 0.07 \mathrm{~mm}$

$T_{\min }=0.713, T_{\max }=0.747$

20409 measured reflections

5826 independent reflections

4937 reflections with $I>2 \sigma(I)$

$R_{\text {int }}=0.030$

$\theta_{\text {max }}=34.0^{\circ}, \theta_{\min }=2.8^{\circ}$

$h=-11 \rightarrow 13$

$k=-14 \rightarrow 14$

$l=-15 \rightarrow 15$ 


\section{Refinement \\ Refinement on $F^{2}$ \\ Least-squares matrix: full $R\left[F^{2}>2 \sigma\left(F^{2}\right)\right]=$ 0.040 \\ $w R\left(F^{2}\right)=0.105$ \\ $S=1.06$ \\ 5826 reflections \\ 244 parameters \\ Special details}

0 restraints

Primary atom site location: iterative

Hydrogen site location: difference Fourier map

H_atoms_treated_by_a_mixture_of_independent_and_constrained_refinem $w=1 /\left[\sigma^{2}\left(F_{\mathrm{o}}^{2}\right)+(0.039 \overline{1} P)^{2}+0 . \overline{2} 95 \overline{1} P\right]$

where $P=\left(F_{\mathrm{o}}^{2}+2 F_{\mathrm{c}}^{2}\right) / 3$

$(\Delta / \sigma)_{\max }<0.001$

$\Delta \rho_{\max }=0.48 \mathrm{e} \AA^{-3}$

$\Delta \rho_{\min }=-0.27$ e $\AA^{-3}$

Geometry. All esds (except the esd in the dihedral angle between two 1.s. planes) are estimated using the full covariance matrix. The cell esds are taken into account individually in the estimation of esds in distances, angles and torsion angles; correlations between esds in cell parameters are only used when they are defined by crystal symmetry. An approximate (isotropic) treatment of cell esds is used for estimating esds involving l.s. planes.

Fractional atomic coordinates and isotropic or equivalent isotropic displacement parameters $\left(\AA^{2}\right)$

\begin{tabular}{lllll}
\hline & $x$ & $y$ & $z$ & $U_{\text {iso }} / U_{\text {eq }}$ \\
\hline Si1 & $0.69854(3)$ & $0.37535(3)$ & $0.34297(3)$ & $0.01325(7)$ \\
O1 & $0.92417(10)$ & $0.22237(9)$ & $0.28540(9)$ & $0.02194(16)$ \\
C1 & $0.86366(11)$ & $0.32897(11)$ & $0.24356(10)$ & $0.01447(16)$ \\
C2 & $0.93945(12)$ & $0.38813(13)$ & $0.13924(11)$ & $0.01886(18)$ \\
C3 & $1.07424(14)$ & $0.32450(15)$ & $0.10108(13)$ & $0.0250(2)$ \\
H3A & $1.182(2)$ & $0.410(2)$ & $0.1241(18)$ & $0.035(4)^{*}$ \\
H3B & $1.068(2)$ & $0.282(2)$ & $0.0052(19)$ & $0.037(4)^{*}$ \\
C4 & $1.04574(14)$ & $0.20014(14)$ & $0.19082(13)$ & $0.0241(2)$ \\
H4A & $1.003(2)$ & $0.091(2)$ & $0.1329(18)$ & $0.037(4)^{*}$ \\
H4B & $1.143(2)$ & $0.213(2)$ & $0.2498(17)$ & $0.034(4)^{*}$ \\
C5 & $0.50711(11)$ & $0.19477(11)$ & $0.29840(10)$ & $0.01461(16)$ \\
C6 & $0.44747(12)$ & $0.11776(12)$ & $0.15886(10)$ & $0.01701(17)$ \\
H6 & $0.4976(18)$ & $0.1611(17)$ & $0.0857(15)$ & $0.021(3)^{*}$ \\
C7 & $0.31274(12)$ & $-0.02389(13)$ & $0.12562(11)$ & $0.01983(19)$ \\
H7 & $0.276(2)$ & $-0.0716(19)$ & $0.0290(17)$ & $0.030(4)^{*}$ \\
C8 & $0.23486(13)$ & $-0.09184(13)$ & $0.23187(13)$ & $0.0221(2)$ \\
H8 & $0.1407(19)$ & $-0.1954(19)$ & $0.2088(16)$ & $0.028(4)^{*}$ \\
C9 & $0.28960(13)$ & $-0.01617(13)$ & $0.37054(12)$ & $0.0235(2)$ \\
H9 & $0.232(2)$ & $-0.063(2)$ & $0.4496(17)$ & $0.034(4)^{*}$ \\
C10 & $0.42448(12)$ & $0.12571(13)$ & $0.40346(11)$ & $0.01958(18)$ \\
H10 & $0.4595(19)$ & $0.1741(18)$ & $0.5007(16)$ & $0.025(4)^{*}$ \\
C11 & $0.68003(11)$ & $0.55198(11)$ & $0.28740(10)$ & $0.01557(16)$ \\
C12 & $0.54668(13)$ & $0.54371(13)$ & $0.20270(11)$ & $0.01901(18)$ \\
H12 & $0.4588(18)$ & $0.4466(18)$ & $0.1690(15)$ & $0.021(3)^{*}$ \\
C13 & $0.53731(15)$ & $0.67991(14)$ & $0.16669(12)$ & $0.0236(2)$ \\
H13 & $0.447(2)$ & $0.6702(19)$ & $0.1106(17)$ & $0.029(4)^{*}$ \\
C14 & $0.66219(15)$ & $0.82626(14)$ & $0.21337(12)$ & $0.0239(2)$ \\
H14 & $0.6560(19)$ & $0.9203(19)$ & $0.1908(17)$ & $0.029(4)^{*}$ \\
C15 & $0.79733(14)$ & $0.83698(13)$ & $0.29579(13)$ & $0.0234(2)$ \\
C16 & $0.80543(13)$ & $0.70145(12)$ & $0.33290(12)$ & $0.02067(19)$ \\
& & & &
\end{tabular}




\begin{tabular}{lllll} 
H16 & $0.902(2)$ & $0.7112(19)$ & $0.3922(17)$ & $0.029(4)^{*}$ \\
C17 & $0.76169(14)$ & $0.42855(14)$ & $0.53383(11)$ & $0.02152(19)$ \\
H17A & $0.779(2)$ & $0.341(2)$ & $0.5607(19)$ & $0.042(5)^{*}$ \\
H17B & $0.678(2)$ & $0.459(2)$ & $0.5845(19)$ & $0.041(5)^{*}$ \\
H15 & $0.886(2)$ & $0.9381(19)$ & $0.3294(16)$ & $0.028(4)^{*}$ \\
H17C & $0.863(2)$ & $0.524(2)$ & $0.5574(19)$ & $0.045(5)^{*}$ \\
H2 & $0.9161(19)$ & $0.4673(19)$ & $0.1014(16)$ & $0.028(4)^{*}$ \\
\hline
\end{tabular}

Atomic displacement parameters $\left(\AA^{2}\right)$

\begin{tabular}{lllllll}
\hline & $U^{11}$ & $U^{22}$ & $U^{33}$ & $U^{12}$ & $U^{13}$ & $U^{23}$ \\
\hline Si1 & $0.01095(11)$ & $0.01317(12)$ & $0.01416(12)$ & $0.00328(9)$ & $0.00118(8)$ & $0.00181(9)$ \\
O1 & $0.0202(3)$ & $0.0199(3)$ & $0.0313(4)$ & $0.0113(3)$ & $0.0083(3)$ & $0.0103(3)$ \\
C1 & $0.0112(3)$ & $0.0134(4)$ & $0.0177(4)$ & $0.0040(3)$ & $0.0002(3)$ & $0.0020(3)$ \\
C2 & $0.0156(4)$ & $0.0227(5)$ & $0.0198(4)$ & $0.0084(3)$ & $0.0040(3)$ & $0.0054(4)$ \\
C3 & $0.0176(4)$ & $0.0309(6)$ & $0.0272(5)$ & $0.0104(4)$ & $0.0078(4)$ & $0.0051(4)$ \\
C4 & $0.0194(4)$ & $0.0225(5)$ & $0.0318(6)$ & $0.0116(4)$ & $0.0051(4)$ & $0.0019(4)$ \\
C5 & $0.0114(3)$ & $0.0150(4)$ & $0.0170(4)$ & $0.0042(3)$ & $0.0020(3)$ & $0.0036(3)$ \\
C6 & $0.0144(4)$ & $0.0176(4)$ & $0.0169(4)$ & $0.0044(3)$ & $0.0023(3)$ & $0.0015(3)$ \\
C7 & $0.0150(4)$ & $0.0186(4)$ & $0.0217(4)$ & $0.0044(3)$ & $0.0004(3)$ & $-0.0014(3)$ \\
C8 & $0.0158(4)$ & $0.0165(4)$ & $0.0307(5)$ & $0.0030(3)$ & $0.0023(4)$ & $0.0036(4)$ \\
C9 & $0.0198(4)$ & $0.0210(5)$ & $0.0270(5)$ & $0.0019(4)$ & $0.0052(4)$ & $0.0088(4)$ \\
C10 & $0.0171(4)$ & $0.0197(4)$ & $0.0192(4)$ & $0.0026(3)$ & $0.0025(3)$ & $0.0056(4)$ \\
C11 & $0.0138(4)$ & $0.0149(4)$ & $0.0178(4)$ & $0.0055(3)$ & $0.0038(3)$ & $0.0024(3)$ \\
C12 & $0.0176(4)$ & $0.0185(4)$ & $0.0214(4)$ & $0.0071(3)$ & $0.0017(3)$ & $0.0046(3)$ \\
C13 & $0.0252(5)$ & $0.0249(5)$ & $0.0249(5)$ & $0.0127(4)$ & $0.0020(4)$ & $0.0084(4)$ \\
C14 & $0.0303(5)$ & $0.0206(5)$ & $0.0265(5)$ & $0.0133(4)$ & $0.0083(4)$ & $0.0100(4)$ \\
C15 & $0.0243(5)$ & $0.0159(4)$ & $0.0292(5)$ & $0.0058(4)$ & $0.0070(4)$ & $0.0055(4)$ \\
C16 & $0.0169(4)$ & $0.0169(4)$ & $0.0265(5)$ & $0.0043(3)$ & $0.0019(4)$ & $0.0042(4)$ \\
C17 & $0.0205(4)$ & $0.0237(5)$ & $0.0161(4)$ & $0.0040(4)$ & $0.0001(3)$ & $0.0025(4)$ \\
& & & & & & \\
\hline
\end{tabular}

Geometric parameters $\left(\AA,{ }^{\circ}\right)$

\begin{tabular}{llll}
\hline Si1-C1 & $1.8743(10)$ & $\mathrm{C} 8-\mathrm{H} 8$ & $0.998(16)$ \\
$\mathrm{Si} 1-\mathrm{C} 5$ & $1.8720(9)$ & $\mathrm{C} 8-\mathrm{C} 9$ & $1.3854(17)$ \\
$\mathrm{S} i 1-\mathrm{C} 11$ & $1.8715(10)$ & $\mathrm{C} 9-\mathrm{H} 9$ & $1.023(16)$ \\
$\mathrm{Si} 1-\mathrm{C} 17$ & $1.8591(11)$ & $\mathrm{C} 9-\mathrm{C} 10$ & $1.3944(15)$ \\
$\mathrm{O} 1-\mathrm{C} 1$ & $1.3897(12)$ & $\mathrm{C} 10-\mathrm{H} 10$ & $0.960(15)$ \\
$\mathrm{O} 1-\mathrm{C} 4$ & $1.4603(13)$ & $\mathrm{C} 11-\mathrm{C} 12$ & $1.3988(14)$ \\
$\mathrm{C} 1-\mathrm{C} 2$ & $1.3356(14)$ & $\mathrm{C} 11-\mathrm{C} 16$ & $1.4050(14)$ \\
$\mathrm{C} 2-\mathrm{C} 3$ & $1.5082(15)$ & $\mathrm{C} 12-\mathrm{H} 12$ & $0.944(15)$ \\
$\mathrm{C} 2-\mathrm{H} 2$ & $0.961(16)$ & $\mathrm{C} 12-\mathrm{C} 13$ & $1.3962(15)$ \\
$\mathrm{C} 3-\mathrm{H} 3 \mathrm{~A}$ & $0.981(17)$ & $\mathrm{C} 13-\mathrm{H} 13$ & $0.928(16)$ \\
$\mathrm{C} 3-\mathrm{H} 3 \mathrm{~B}$ & $0.933(18)$ & $\mathrm{C} 13-\mathrm{C} 14$ & $1.3861(17)$ \\
$\mathrm{C} 3-\mathrm{C} 4$ & $1.5398(18)$ & $\mathrm{C} 14-\mathrm{H} 14$ & $0.953(16)$ \\
$\mathrm{C} 4-\mathrm{H} 4 \mathrm{~A}$ & $0.982(17)$ & $\mathrm{C} 14-\mathrm{C} 15$ & $1.3909(17)$ \\
$\mathrm{C} 4-\mathrm{H} 4 \mathrm{~B}$ & $0.987(17)$ & $\mathrm{C} 15-\mathrm{C} 16$ & $1.3911(15)$ \\
$\mathrm{C} 5-\mathrm{C} 6$ & $1.4023(14)$ & $\mathrm{C} 15-\mathrm{H} 15$ & $0.969(16)$
\end{tabular}




\begin{tabular}{|c|c|c|c|}
\hline $\mathrm{C} 5-\mathrm{C} 10$ & $1.4014(13)$ & $\mathrm{C} 16-\mathrm{H} 16$ & $0.990(16)$ \\
\hline C6-H6 & $0.934(15)$ & C17-H17A & $0.950(18)$ \\
\hline $\mathrm{C} 6-\mathrm{C} 7$ & $1.3926(14)$ & C17-H17B & 0.975 (19) \\
\hline $\mathrm{C} 7-\mathrm{H} 7$ & $0.956(16)$ & $\mathrm{C} 17-\mathrm{H} 17 \mathrm{C}$ & $0.992(19)$ \\
\hline $\mathrm{C} 7-\mathrm{C} 8$ & $1.3919(16)$ & & \\
\hline $\mathrm{C} 5-\mathrm{Si} 1-\mathrm{C} 1$ & $108.44(4)$ & $\mathrm{C} 7-\mathrm{C} 8-\mathrm{H} 8$ & $120.4(9)$ \\
\hline $\mathrm{C} 11-\mathrm{Si} 1-\mathrm{C} 1$ & $105.50(4)$ & $\mathrm{C} 9-\mathrm{C} 8-\mathrm{C} 7$ & $119.76(10)$ \\
\hline $\mathrm{C} 11-\mathrm{Si} 1-\mathrm{C} 5$ & $113.08(4)$ & $\mathrm{C} 9-\mathrm{C} 8-\mathrm{H} 8$ & $119.8(9)$ \\
\hline $\mathrm{C} 17-\mathrm{Si} 1-\mathrm{C} 1$ & $109.26(5)$ & $\mathrm{C} 8-\mathrm{C} 9-\mathrm{H} 9$ & $120.5(9)$ \\
\hline $\mathrm{C} 17-\mathrm{Si} 1-\mathrm{C} 5$ & $110.48(5)$ & $\mathrm{C} 8-\mathrm{C} 9-\mathrm{C} 10$ & $120.10(10)$ \\
\hline $\mathrm{C} 17-\mathrm{Si} 1-\mathrm{C} 11$ & $109.89(5)$ & $\mathrm{C} 10-\mathrm{C} 9-\mathrm{H} 9$ & $119.4(9)$ \\
\hline $\mathrm{C} 1-\mathrm{O} 1-\mathrm{C} 4$ & $107.48(8)$ & $\mathrm{C} 5-\mathrm{C} 10-\mathrm{H} 10$ & $121.0(9)$ \\
\hline $\mathrm{O} 1-\mathrm{C} 1-\mathrm{Si} 1$ & $117.13(7)$ & $\mathrm{C} 9-\mathrm{C} 10-\mathrm{C} 5$ & $121.24(10)$ \\
\hline $\mathrm{C} 2-\mathrm{C} 1-\mathrm{Si} 1$ & $129.71(8)$ & $\mathrm{C} 9-\mathrm{C} 10-\mathrm{H} 10$ & $117.7(9)$ \\
\hline $\mathrm{C} 2-\mathrm{C} 1-\mathrm{O} 1$ & $113.10(9)$ & $\mathrm{C} 12-\mathrm{C} 11-\mathrm{Si} 1$ & $123.40(7)$ \\
\hline $\mathrm{C} 1-\mathrm{C} 2-\mathrm{C} 3$ & $110.25(9)$ & $\mathrm{C} 12-\mathrm{C} 11-\mathrm{C} 16$ & $117.71(9)$ \\
\hline $\mathrm{C} 1-\mathrm{C} 2-\mathrm{H} 2$ & $123.6(9)$ & $\mathrm{C} 16-\mathrm{C} 11-\mathrm{Si} 1$ & $118.89(8)$ \\
\hline $\mathrm{C} 3-\mathrm{C} 2-\mathrm{H} 2$ & $126.0(9)$ & $\mathrm{C} 11-\mathrm{C} 12-\mathrm{H} 12$ & $121.5(9)$ \\
\hline $\mathrm{C} 2-\mathrm{C} 3-\mathrm{H} 3 \mathrm{~A}$ & $111.2(10)$ & $\mathrm{C} 13-\mathrm{C} 12-\mathrm{C} 11$ & $121.09(10)$ \\
\hline $\mathrm{C} 2-\mathrm{C} 3-\mathrm{H} 3 \mathrm{~B}$ & $112.3(11)$ & $\mathrm{C} 13-\mathrm{C} 12-\mathrm{H} 12$ & $117.4(9)$ \\
\hline $\mathrm{C} 2-\mathrm{C} 3-\mathrm{C} 4$ & $101.51(9)$ & $\mathrm{C} 12-\mathrm{C} 13-\mathrm{H} 13$ & $118.8(10)$ \\
\hline $\mathrm{H} 3 \mathrm{~A}-\mathrm{C} 3-\mathrm{H} 3 \mathrm{~B}$ & $105.5(14)$ & $\mathrm{C} 14-\mathrm{C} 13-\mathrm{C} 12$ & $120.16(10)$ \\
\hline $\mathrm{C} 4-\mathrm{C} 3-\mathrm{H} 3 \mathrm{~A}$ & $112.9(10)$ & $\mathrm{C} 14-\mathrm{C} 13-\mathrm{H} 13$ & $121.0(10)$ \\
\hline $\mathrm{C} 4-\mathrm{C} 3-\mathrm{H} 3 \mathrm{~B}$ & $113.7(11)$ & $\mathrm{C} 13-\mathrm{C} 14-\mathrm{H} 14$ & $120.8(10)$ \\
\hline $\mathrm{O} 1-\mathrm{C} 4-\mathrm{C} 3$ & $106.70(8)$ & $\mathrm{C} 13-\mathrm{C} 14-\mathrm{C} 15$ & $119.80(10)$ \\
\hline $\mathrm{O} 1-\mathrm{C} 4-\mathrm{H} 4 \mathrm{~A}$ & $108.8(10)$ & $\mathrm{C} 15-\mathrm{C} 14-\mathrm{H} 14$ & $119.4(10)$ \\
\hline $\mathrm{O} 1-\mathrm{C} 4-\mathrm{H} 4 \mathrm{~B}$ & $107.0(10)$ & $\mathrm{C} 14-\mathrm{C} 15-\mathrm{C} 16$ & $119.92(10)$ \\
\hline $\mathrm{C} 3-\mathrm{C} 4-\mathrm{H} 4 \mathrm{~A}$ & $111.5(10)$ & $\mathrm{C} 14-\mathrm{C} 15-\mathrm{H} 15$ & $121.1(9)$ \\
\hline $\mathrm{C} 3-\mathrm{C} 4-\mathrm{H} 4 \mathrm{~B}$ & $114.2(10)$ & $\mathrm{C} 16-\mathrm{C} 15-\mathrm{H} 15$ & $119.0(9)$ \\
\hline $\mathrm{H} 4 \mathrm{~A}-\mathrm{C} 4-\mathrm{H} 4 \mathrm{~B}$ & $108.4(14)$ & $\mathrm{C} 11-\mathrm{C} 16-\mathrm{H} 16$ & $119.7(9)$ \\
\hline $\mathrm{C} 6-\mathrm{C} 5-\mathrm{Si} 1$ & $121.29(7)$ & $\mathrm{C} 15-\mathrm{C} 16-\mathrm{C} 11$ & $121.32(10)$ \\
\hline $\mathrm{C} 10-\mathrm{C} 5-\mathrm{Si} 1$ & $120.94(7)$ & $\mathrm{C} 15-\mathrm{C} 16-\mathrm{H} 16$ & $118.9(9)$ \\
\hline $\mathrm{C} 10-\mathrm{C} 5-\mathrm{C} 6$ & $117.64(9)$ & Si1-C17-H17A & $109.9(11)$ \\
\hline $\mathrm{C} 5-\mathrm{C} 6-\mathrm{H} 6$ & $120.3(9)$ & Si1-C17-H17B & $108.7(11)$ \\
\hline $\mathrm{C} 7-\mathrm{C} 6-\mathrm{C} 5$ & $121.26(9)$ & $\mathrm{Si} 1-\mathrm{C} 17-\mathrm{H} 17 \mathrm{C}$ & $110.5(11)$ \\
\hline $\mathrm{C} 7-\mathrm{C} 6-\mathrm{H} 6$ & $118.4(9)$ & $\mathrm{H} 17 \mathrm{~A}-\mathrm{C} 17-\mathrm{H} 17 \mathrm{~B}$ & $112.2(15)$ \\
\hline $\mathrm{C} 6-\mathrm{C} 7-\mathrm{H} 7$ & $118.2(10)$ & $\mathrm{H} 17 \mathrm{~A}-\mathrm{C} 17-\mathrm{H} 17 \mathrm{C}$ & $109.4(15)$ \\
\hline $\mathrm{C} 8-\mathrm{C} 7-\mathrm{C} 6$ & $119.98(10)$ & $\mathrm{H} 17 \mathrm{~B}-\mathrm{C} 17-\mathrm{H} 17 \mathrm{C}$ & $106.1(15)$ \\
\hline $\mathrm{C} 8-\mathrm{C} 7-\mathrm{H} 7$ & $121.8(10)$ & & \\
\hline $\mathrm{Si} 1-\mathrm{C} 1-\mathrm{C} 2-\mathrm{C} 3$ & $175.12(8)$ & $\mathrm{C} 6-\mathrm{C} 7-\mathrm{C} 8-\mathrm{C} 9$ & $1.46(16)$ \\
\hline $\mathrm{Si} 1-\mathrm{C} 5-\mathrm{C} 6-\mathrm{C} 7$ & $174.69(8)$ & $\mathrm{C} 7-\mathrm{C} 8-\mathrm{C} 9-\mathrm{C} 10$ & $-1.42(17)$ \\
\hline $\mathrm{Si} 1-\mathrm{C} 5-\mathrm{C} 10-\mathrm{C} 9$ & $-174.66(9)$ & $\mathrm{C} 8-\mathrm{C} 9-\mathrm{C} 10-\mathrm{C} 5$ & $0.07(17)$ \\
\hline $\mathrm{Si} 1-\mathrm{C} 11-\mathrm{C} 12-\mathrm{C} 13$ & $178.63(8)$ & $\mathrm{C} 10-\mathrm{C} 5-\mathrm{C} 6-\mathrm{C} 7$ & $-1.15(15)$ \\
\hline $\mathrm{Si} 1-\mathrm{C} 11-\mathrm{C} 16-\mathrm{C} 15$ & $-179.42(8)$ & $\mathrm{C} 11-\mathrm{Si} 1-\mathrm{C} 1-\mathrm{O} 1$ & $167.77(7)$ \\
\hline $\mathrm{O} 1-\mathrm{C} 1-\mathrm{C} 2-\mathrm{C} 3$ & $-2.00(12)$ & $\mathrm{C} 11-\mathrm{Si} 1-\mathrm{C} 1-\mathrm{C} 2$ & $-9.26(11)$ \\
\hline $\mathrm{C} 1-\mathrm{Si} 1-\mathrm{C} 5-\mathrm{C} 6$ & $-51.23(9)$ & $\mathrm{C} 11-\mathrm{Si} 1-\mathrm{C} 5-\mathrm{C} 6$ & $65.41(9)$ \\
\hline
\end{tabular}




\begin{tabular}{|c|c|c|c|}
\hline $\mathrm{C} 1-\mathrm{Si} 1-\mathrm{C} 5-\mathrm{C} 10$ & $124.48(8)$ & $\mathrm{C} 11-\mathrm{Si} 1-\mathrm{C} 5-\mathrm{C} 10$ & $-118.88(9)$ \\
\hline $\mathrm{C} 1-\mathrm{Si} 1-\mathrm{C} 11-\mathrm{C} 12$ & $109.11(9)$ & $\mathrm{C} 11-\mathrm{C} 12-\mathrm{C} 13-\mathrm{C} 14$ & $0.82(17)$ \\
\hline $\mathrm{C} 1-\mathrm{Si} 1-\mathrm{C} 11-\mathrm{C} 16$ & $-71.17(9)$ & $\mathrm{C} 12-\mathrm{C} 11-\mathrm{C} 16-\mathrm{C} 15$ & $0.31(15)$ \\
\hline $\mathrm{C} 1-\mathrm{O} 1-\mathrm{C} 4-\mathrm{C} 3$ & $8.95(11)$ & $\mathrm{C} 12-\mathrm{C} 13-\mathrm{C} 14-\mathrm{C} 15$ & $0.27(17)$ \\
\hline $\mathrm{C} 1-\mathrm{C} 2-\mathrm{C} 3-\mathrm{C} 4$ & $7.17(12)$ & $\mathrm{C} 13-\mathrm{C} 14-\mathrm{C} 15-\mathrm{C} 16$ & $-1.03(17)$ \\
\hline $\mathrm{C} 2-\mathrm{C} 3-\mathrm{C} 4-\mathrm{O} 1$ & $-9.52(11)$ & $\mathrm{C} 14-\mathrm{C} 15-\mathrm{C} 16-\mathrm{C} 11$ & $0.74(17)$ \\
\hline $\mathrm{C} 4-\mathrm{O} 1-\mathrm{C} 1-\mathrm{Si} 1$ & $177.92(7)$ & $\mathrm{C} 16-\mathrm{C} 11-\mathrm{C} 12-\mathrm{C} 13$ & $-1.09(15)$ \\
\hline $\mathrm{C} 4-\mathrm{O} 1-\mathrm{C} 1-\mathrm{C} 2$ & $-4.57(12)$ & $\mathrm{C} 17-\mathrm{Si} 1-\mathrm{C} 1-\mathrm{O} 1$ & $49.68(8)$ \\
\hline $\mathrm{C} 5-\mathrm{Si} 1-\mathrm{C} 1-\mathrm{O} 1$ & $-70.80(8)$ & $\mathrm{C} 17-\mathrm{Si} 1-\mathrm{C} 1-\mathrm{C} 2$ & $-127.35(10)$ \\
\hline $\mathrm{C} 5-\mathrm{Si} 1-\mathrm{C} 1-\mathrm{C} 2$ & $112.17(10)$ & $\mathrm{C} 17-\mathrm{Si} 1-\mathrm{C} 5-\mathrm{C} 6$ & $-170.95(8)$ \\
\hline $\mathrm{C} 5-\mathrm{Si} 1-\mathrm{C} 11-\mathrm{C} 12$ & $-9.26(10)$ & $\mathrm{C} 17-\mathrm{Si} 1-\mathrm{C} 5-\mathrm{C} 10$ & $4.76(10)$ \\
\hline $\mathrm{C} 5-\mathrm{Si} 1-\mathrm{C} 11-\mathrm{C} 16$ & $170.46(8)$ & $\mathrm{C} 17-\mathrm{Si} 1-\mathrm{C} 11-\mathrm{C} 12$ & $-133.23(9)$ \\
\hline $\mathrm{C} 5-\mathrm{C} 6-\mathrm{C} 7-\mathrm{C} 8$ & $-0.15(16)$ & $\mathrm{C} 17-\mathrm{Si} 1-\mathrm{C} 11-\mathrm{C} 16$ & $46.49(9)$ \\
\hline $\mathrm{C} 6-\mathrm{C} 5-\mathrm{C} 10-\mathrm{C} 9$ & $1.20(15)$ & & \\
\hline
\end{tabular}

Hydrogen-bond geometry $\left(\AA,{ }^{\circ}\right)$

\begin{tabular}{lllll}
\hline$D-\mathrm{H} \cdots A$ & $D-\mathrm{H}$ & $\mathrm{H} \cdots A$ & $D \cdots A$ & $D-\mathrm{H} \cdots A$ \\
\hline $\mathrm{C} 15-\mathrm{H} 15 \cdots \mathrm{O} 1^{\mathrm{i}}$ & $0.969(16)$ & $2.640(16)$ & $3.3422(13)$ & $129.6(12)$ \\
$\mathrm{C} 17-\mathrm{H} 17 C \cdots \mathrm{O} 1^{\mathrm{ii}}$ & $0.992(19)$ & $2.584(19)$ & $3.5168(14)$ & $156.5(15)$ \\
\hline
\end{tabular}

Symmetry codes: (i) $x, y+1, z$; (ii) $-x+2,-y+1,-z+1$.

Selected geometric parameters for compound $1(\AA, \circ)$

\begin{tabular}{llll}
\hline $\mathrm{Si} 1-\mathrm{C} 1$ & $1.8742(3)$ & $\mathrm{C} 1-\mathrm{Si} 1-\mathrm{C} 5$ & $108.189(12)$ \\
$\mathrm{Si} 1-\mathrm{C} 5$ & $1.8693(3)$ & $\mathrm{C} 1-\mathrm{Si} 1-\mathrm{C} 9$ & $106.801(14)$ \\
$\mathrm{Si} 1-\mathrm{C} 9$ & $1.8631(3)$ & $\mathrm{C} 1-\mathrm{Si} 1-\mathrm{C} 10$ & $109.191(13)$ \\
$\mathrm{Si} 1-\mathrm{C} 10$ & $1.8579(3)$ & $\mathrm{C} 5-\mathrm{Si} 1-\mathrm{C} 9$ & $110.770(15)$ \\
& & $\mathrm{C} 5-\mathrm{Si} 1-\mathrm{C} 10$ & $108.128(13)$ \\
$\mathrm{C} 1-\mathrm{C} 2$ & $1.3370(4)$ & $\mathrm{C} 9-\mathrm{Si} 1-\mathrm{C} 10$ & $113.628(16)$ \\
$\mathrm{C} 3-\mathrm{C} 4$ & $1.5331(5)$ & & \\
$\mathrm{C} 5-\mathrm{C} 6$ & $1.3409(4)$ & & \\
$\mathrm{C} 7-\mathrm{C} 8$ & $1.5298(5)$ & & \\
\hline
\end{tabular}

